\title{
Haushaltsüberschüsse und ihre Verwendung: Wiedergewinnung staatlicher Handlungsfähigkeit?
}

von Lukas Haffert und Philip Mehrtens

Eine weitverbreitete Rechtfertigung für politisch unpopuläre Haushaltskonsolidierungen lautet, diese erlaubten eine Wiedergewinnung staatlicher Handlungsfähigkeit. Auch in Deutschland verbinden sich mit den angekündigten Überschüssen im Bundeshaushalt große Hoffnungen auf größere Investitionen in Bildung, Familien und Infrastruktur. Diese Studie diskutiert diese "progressive Konsolidierungsthese" aus theoretischer Perspektive und untersucht danach empirisch die Entwicklung der Staatstätigkeit in sechs Ländern mit dauerhaften Haushaltsüberschüssen. Sie kommt zu dem Schluss, dass sich die Hoffnungen der progressiven Konsolidierungsthese häufig nicht erfüllen, weil Überschüsse zwar fiskalische Spielräume erhöhen, zugleich aber politische Spielräume reduzieren. Während Überschüsse spürbare Steuersenkungen ermöglichten, profitierten die öffentlichen Investitionen kaum von ihnen.

Politically unpopular austerity measures are often justified by arguing that successful fiscal consolidation will lead to a strengthening of the fiscal capacity of the state. For example, many observers of German fiscal policy hope that federal budget surpluses will lead to greater public investment in education, families and public infrastructure. In this paper we discuss these predictions theoretically and test their empirical validity by analyzing public expenditure in six countries with sustained budget surpluses. We show that progressive hopes often proved to be unfounded. While consolidation efforts increased fiscal space, they reduced political space at the same time. Surpluses were predominantly used for cutting taxes and not for increasing public investment again.

\section{Einleitung ${ }^{1}$}

„Wer zukünftig einen handlungsfähigen Staat will, wer die Gestaltungsfähigkeit der Politik und nachfolgender Parlamentariergenerationen erhöhen will, der muss dafür sorgen, dass Schuldenstand und Zinslast reduziert werden. Ein handlungsfähiger Staat braucht langfristig tragfähige öffentliche Finanzen. ${ }^{\text {"2 }}$

1 Wir danken den zwei anonymen Gutachtern der ZSE für ihre hilfreichen Kommentare.

2 Deutscher Bundestag: Plenarprotokoll der 225. Sitzung der 16. Wahlperiode (29.05.2009), hier 24866. 
Mit diesem Argument warb der damalige Bundesfinanzminister Peer Steinbrück im Mai 2009 im deutschen Bundestag für die Verankerung der sogenannten Schuldenbremse im Grundgesetz. Diese Debatte fand damals vor dem Hintergrund einer sich wegen der Weltfinanzkrise rapide verschlechternden Haushaltslage statt. Heute steht die Bundesrepublik dagegen an der Schwelle zu Haushaltsüberschüssen. Dies wirft die Frage auf, ob solche Überschüsse tatsächlich mit der von Steinbrück in Aussicht gestellten Wiedergewinnung staatlicher Handlungsfähigkeit verbunden sind.

Konkrete Prognosen für die deutsche Fiskalpolitik der kommenden Jahre sind naturgemäß schwer zu treffen, weil sowohl über die zu erwartenden politischen Entscheidungen als auch über die makroökonomischen Rahmenbedingungen erhebliche Unsicherheiten bestehen. ${ }^{3}$ Sinnvoller erscheint es daher, die theoretische und empirische Validität von Steinbrücks Argument am Beispiel anderer Länder in den Jahren vor der jüngsten Weltfinanzkrise zu untersuchen. Steinbrück steht mit seiner These über den Zusammenhang von Konsolidierungen und staatlicher Gestaltungsfähigkeit keineswegs allein. So war dieser Ansatz beispielsweise in den 1990er-Jahren unter Vertretern eines sogenannten dritten Weges, wie Bill Clinton und Göran Persson, weit verbreitet. ${ }^{4}$

Er wird im Folgenden als progressive Konsolidierungsthese bezeichnet. Dabei müssen progressive Konsolidierungen nicht zwangsläufig von progressiven Regierungen betrieben werden. Was sie ausmacht, ist vielmehr ihre progressive Zieldefinition. Diese könnte unter der paradoxen Überschrift Handlungsfähigkeit durch Austerität zusammengefasst werden. Aus der Tatsache, dass Schulden und Defizite die Handlungsfähigkeit des Staates immer weiter einschränken, folgern die Befürworter progressiver Konsolidierungen im Umkehrschluss, dass Überschüsse und der Abbau der Staatsverschuldung die Gestaltungsspielräume der Politik wieder erhöhen. Haushaltskonsolidierungen sind deshalb kein Zweck an sich, sondern ein Mittel zur Wiedergewinnung staatlicher Handlungsfähigkeit. Sie sind nur ein erster Schritt, der die nötigen Ressourcen für die eigentlichen Politikziele erbringen soll.

Der Erfolg einer progressiven Haushaltskonsolidierung lässt sich deshalb erst mit einigen Jahren Abstand beurteilen. Ob eine Konsolidierung ihren Zweck erfüllt, zeigt sich nicht bereits an einem Ausgleich des Budgetsaldos. Erst wenn die

3 Wagschal, U.: Quo vadis Verschuldung? Szenarien der Haushaltspolitik, in: Zeitschrift für Staats- und Europawissenschaften, 11/2 (2013), 213-238.

4 Romano, F.: Clinton and Blair, The Political Economy of the Third Way, London, 2006; Persson, G. (mit Kask, J.-P.): Den som är satt i skuld är icke fri, Stockholm, 1997. 
„,schwarze Null“ tatsächlich mit einem Wiederausbau staatlicher Gestaltungsausgaben verbunden ist, ist eine progressive Konsolidierung gelungen. Die umfangreiche Literatur zu den Determinanten erfolgreicher Haushaltskonsolidierungen ${ }^{5}$ ist daher zur Beurteilung der progressiven Konsolidierungsthese nur begrenzt geeignet, da sich diese Studien auf die Entstehung von Überschüssen konzentrieren, nicht aber auf die politische Verwendung des neu gewonnenen fiskalischen Spielraums.

Die vorliegende Arbeit untersucht die Verwendung von Haushaltsüberschüssen. Dazu entwickelt sie zunächst die empirischen Vorhersagen der progressiven Konsolidierungsthese und vergleicht sie mit den Prognosen einer historisch-institutionalistischen Perspektive, die die erheblichen Kontinuitäten und Pfadabhängigkeiten zwischen der Politik während und nach einer Konsolidierung betont. Danach analysiert die Arbeit die Entwicklung der Staatstätigkeit in Ländern mit dauerhaften Haushaltsüberschüssen. Diese verfügten über erhebliche fiskalische Handlungsspielräume und sind somit Most-likely Cases für die progressive Konsolidierungsthese. Die Analyse zeigt, dass sich die fiskalpolitischen Trends der Konsolidierung im Überschuss weiter fortsetzten, anstatt sich, wie von der progressiven Konsolidierungsthese prognostiziert, umzukehren. Die Studie schließt mit einigen Überlegungen zur Übertragbarkeit dieser Ergebnisse auf zukünftige deutsche Haushaltsüberschüsse.

\section{Zwei Sichtweisen auf Haushaltskonsolidierungen und ihre Fol- gen für staatliche Handlungsfähigkeit}

Die Grundidee der progressiven Konsolidierungsthese ist, durch eine Haushaltskonsolidierung die fiskalpolitische Handlungsfähigkeit des Staates zurückzugewinnen. Die dafür notwendigen Kürzungen und Austeritätsmaßnahmen werden als notwendige, aber zeitlich befristete Anpassungen betrachtet, um fiskalische Spielräume zu schaffen. Eine progressive Konsolidierung endet also nicht bereits mit einer Verbesserung der Haushaltslage, sondern umfasst darüber hinaus die Verwendung der gewonnenen Finanzmittel zur Ausweitung gestaltender Staatstätigkeit. Der staatliche Gestaltungsspielraum soll dabei nachhaltig erhöht und nicht bereits nach kurzer Zeit wieder von Sparzwängen eingeschränkt werden. Eine langfristige Bewahrung einer guten Haushaltslage ist somit eine Voraussetzung für den Erfolg einer progressiven Konsolidierung.

5 Für viele andere beispielhaft: Alesina, A./Ardagna, S.: The Design of Fiscal Adjustments, NBER Working Paper 18423, 2012; Wagschal, U./Wenzelburger, G.: Roads to Success: Budget Consolidations in OECD Countries, in: Journal of Public Policy, 28/3 (2008), 309-339. 
Konkret ist ein erstes wichtiges Ziel der Vertreter progressiver Konsolidierungen, den Sozialstaat durch ausgeglichene Haushalte „zukunftsfest“ zu machen, das heißt ihn vor noch tieferen Einschnitten in der Zukunft zu schützen. Auf dieser Ebene wäre eine Konsolidierung also bereits erfolgreich, wenn Überschüsse dazu führten, dass Staatsausgaben weniger stark gekürzt werden als in der Vergangenheit oder in vergleichbaren Ländern mit Defiziten. Darüber hinaus wird in der Regel aber auch angestrebt, durch Überschüsse fiskalische Freiräume für neue, gestaltende Politikinitiativen zu schaffen. Das Ziel ist die Rückkehr zu einer Politik, in der nicht jeder Reformvorschlag automatisch unter Finanzierungsvorbehalt steht. Dieser Anspruch ist schwieriger zu erfüllen, weil er nicht nur eine Verlangsamung, sondern sogar eine Umkehrung fiskalischer Trends verlangt. Ausgabenfelder, die besonders von den Sparmaßnahmen während der Konsolidierung betroffen waren, sollen demnach auch stärker von den neuen fiskalischen Möglichkeiten profitieren. Diese Zielsetzung bezieht sich vor allem auf die Formen der Staatstätigkeit, die sowohl auf die Gestaltung der Zukunft gerichtet sind als auch von der Politik gestaltet werden können, also zugleich gestaltend und gestaltbar sind. Das Ziel der progressiven Konsolidierungsthese im engeren Sinne ist damit vor allem eine Erhöhung der öffentlichen Investitionen.

Die Voraussetzung für beide Ziele progressiver Konsolidierungen sind in jedem Fall ausreichende Steuereinnahmen. Zugleich ist die Steuerpolitik aber selbst ein mögliches Feld staatlicher Gestaltungsambitionen. So können Steuersenkungen eingesetzt werden, um private Investitionen zu stimulieren oder bestimmte gesellschaftliche Gruppen gezielt zu entlasten. Insbesondere Steuersubventionen, wie die steuerliche Begünstigung bestimmter Verhaltensweisen, sind oft ein Substitut für direkte Staatsausgaben.

Offenbar stehen die verschiedenen Ziele progressiver Konsolidierungen in einem Zielkonflikt: Je größer der Teil der Staatsausgaben ist, der vor Kürzungen beschützt werden soll, desto kleiner ist der verfügbare Spielraum für neue Initiativen. Je stärker die Steuern gesenkt werden, desto weniger Mittel stehen für Ausgabensteigerungen zur Verfügung. Unter der Bedingung, dass die gute Haushaltssituation bewahrt wird, muss abgewogen werden, welches Politikziel Priorität genießen soll. Eine weitgehende Revision der Konsolidierungsmaßnahmen sowohl auf der Einnahme- als auch auf der Ausgabenseite würde eine Rückkehr zu defizitfinanzierter Politik bedeuten. Deshalb werden in der Praxis nur bestimmte Politikbereiche von dem zurückgewonnenen fiskalischen Handlungsspielraum profitieren können. 
Konstitutiv für die progressive Konsolidierungsthese ist daher insbesondere die Annahme, dass die Politik nach einer erfolgreichen Haushaltskonsolidierung ohne Restriktionen über neue Ausgabensteigerungen entscheiden und die vorhandenen Zielkonflikte gemäß ihrer politischen Präferenzen lösen könne. Mit anderen Worten: Die progressive Konsolidierungsthese setzt voraus, dass mit dem Nachlassen von fiskalischen Zwängen auch automatisch der politische Handlungsspielraum vorhanden ist, über die neu gewonnenen finanziellen Spielräume frei zu verfügen. Während die Auflösung des beschriebenen Zielkonflikts im Defizit von Sachzwängen diktiert wurde, könne sie im Überschuss wieder in Übereinstimmung mit politischen Zielen und Wünschen erfolgen.

Eine ähnliche Auffassung liegt implizit auch vielen Veröffentlichungen der vergleichenden politischen Ökonomie zugrunde, in denen bei der Betrachtung der Effekte des Budgetsaldos oder der Schuldenquote auf die Staatstätigkeit analytisch nicht zwischen Defiziten und Überschüssen differenziert wird. Die Autoren betrachten diese vielmehr als symmetrisch. Überschüsse stellen demnach ein Spiegelbild von Defiziten dar - nur mit anderem Vorzeichen. Aus dem Befund, dass hohe öffentliche Defizite zu Ausgabenkürzungen führen und dass die Investitionen von der Sparpolitik überproportional stark betroffen sind, ${ }^{6}$ wird dann gefolgert, dass den im Defizit vorgenommenen Kürzungen eine spiegelbildliche Steigerung dieser Ausgaben im Überschuss gegenübersteht. ${ }^{7}$

Eine solcher symmetrischer Wiederanstieg ist jedoch gleichbedeutend mit einem ganz erheblichen Politikwechsel: Die Ausgabenbereiche, die im Defizit besonders stark reduziert wurden, würden demnach besonders von den Überschüssen profitieren, während die Politikfelder, die besonders vor Kürzungen geschützt waren, sich bei der Verteilung der neuen Spielräume kaum durchsetzen könnten. Die Symmetrieannahme kann also nur zutreffen, wenn die Politik die Möglichkeit zu einem solchen fiskalpolitischen Kurswechsel hat.

Diese Voraussetzung wird jedoch weder von Politikern, die ihre Hoffnungen zur Rückgewinnung staatlicher Handlungsfähigkeit auf ausgeglichene Haushalte setzen, noch in wissenschaftlichen Studien, die Überschüsse und Defizite als eine einzige analytische Kategorie fassen, hinreichend berücksichtigt. Dadurch unter-

6 Siehe zum Beispiel Obinger, H.: Die Finanzkrise und die Zukunft des Wohlfahrtsstaates, in: Leviathan, 40/3 (2012), 441-461; Breunig, C./Busemeyer, M.: Fiscal Austerity and the Trade-Off between Public Investment and Social Spending, in: Journal of European Public Policy, 19/6 (2012), 921-938.

7 Siehe zum Beispiel Boix, C.: Political Parties and the Supply Side of the Economy, in: American Journal of Political Science, 41/3 (1997), 814-845. 
schätzen sie die politische Dimension der Konsolidierungspolitik und ihre potentiell beschränkenden Folgewirkungen auf die zukünftige fiskalpolitische Handlungsfähigkeit. Zur Durchsetzung einer nachhaltig wirksamen Haushaltskonsolidierung sind grundlegende politische und institutionelle Wandlungsprozesse notwendig, die das fiskalpolitische Klima, die Interessenlage wichtiger Akteure und das Institutionengefüge in einem Land grundlegend verändern können. ${ }^{8}$

Stellt man diese politische Dimension einer Haushaltskonsolidierung und ihre Folgewirkungen ins Zentrum der Analyse, gelangt man zu einer konkurrierenden Perspektive, die im Folgenden als Pfadabhängigkeitsthese bezeichnet wird. Ihr Ausgangspunkt ist die Überlegung, dass fiskalpolitische Handlungsfähigkeit nicht nur vom gegebenen fiskalischen Handlungsspielraum abhängt, sondern auch vom vorhandenen politischen Handlungsspielraum. Die Verfügbarkeit der notwendigen fiskalischen Ressourcen führt nicht automatisch zu einer Erhöhung der staatlichen Handlungsfähigkeit, wenn gleichzeitig politische Zwänge bestehen, die eine freie Wahl der angestrebten Ziele beinträchtigen. Fiskalpolitische Handlungsfähigkeit der Politik ist demnach erst dann gegeben, wenn sie ihre fiskalpolitischen Ziele frei von fiskalischen und politischen Restriktionen wählen kann.

Die Pfadabhängigkeitsthese beruht auf der Annahme, dass in der Vergangenheit getroffene Entscheidungen die Bedingungen, unter denen Fiskalpolitik in der Gegenwart gemacht wird, entscheidend strukturieren, selbst wenn die ursprünglichen Beweggründe dafür mittlerweile nicht mehr gelten sollten. Die Verwendung fiskalischer Spielräume erfolgt nicht unabhängig von ihrer Entstehung, da die Effekte der für den ausgeglichenen Haushalt nötigen Reformen länger fortwirken und die Politik weiterhin bestimmen. Mit einer Verbesserung der Haushaltslage ist nicht automatisch auch eine Veränderung der politischen Handlungsbedingungen verbunden. Die Fähigkeit der Politik, ihre fiskalischen Ziele frei zu wählen, bleibt demnach auch nach Abschluss der Konsolidierung eng begrenzt und der für eine progressive Konsolidierung notwendige Politikwechsel wird sehr unwahrscheinlich.

8 Lewis, T.: In the Long Run We're All Dead: The Canadian Turn to Fiscal Restraint, Vancouver, 2003, Wenzelburger, G.: Haushaltskonsolidierungen und Reformprozesse, Berlin, 2010; Mehrtens, P.: Staatsschulden und Staatstätigkeit: Zur Transformation der politischen Ökonomie Schwedens, Frankfurt, im Erscheinen. 
Tabelle 1: Konkurrierende Thesen zu den Effekten von Konsolidierungen und Überschüssen

\begin{tabular}{l|l|l}
\hline & $\begin{array}{l}\text { Progressive Konsolidie- } \\
\text { rungsthese }\end{array}$ & Pfadabhängigkeitsthese \\
\hline Effekt von Defiziten & $\begin{array}{l}\text { Reduzieren die Hand- } \\
\text { lungsfähigkeit des Staa- } \\
\text { tes } \\
\text { Fiskalische Effekte } \\
\text { von Konsolidierungen } \\
\text { Erhöhen fiskalische } \\
\text { Spielräume }\end{array}$ & $\begin{array}{l}\text { Reduzieren die Hand- } \\
\text { lungsfähigkeit des Staa- } \\
\text { tes } \\
\text { Erhöhen fiskalische } \\
\text { Spielräume }\end{array}$ \\
$\begin{array}{l}\text { Politische Effekte von } \\
\text { Konsolidierungen }\end{array}$ & $\begin{array}{l}\text { Keine andauernde Be- } \\
\text { schränkung des politi- } \\
\text { schen Spielraums }\end{array}$ & $\begin{array}{l}\text { Erhebliche und andau- } \\
\text { ernde Beschränkung des } \\
\text { politischen Spielraums }\end{array}$ \\
$\begin{array}{l}\text { Effekt von Haushalts- } \\
\text { Ëberschüssen }\end{array}$ & $\begin{array}{l}\text { tische Handlungsfähig- } \\
\text { keit in symmetrischer } \\
\text { Form zu Defiziten }\end{array}$ & $\begin{array}{l}\text { Erhöhen die fiskalpoliti- } \\
\text { sche Handlungsfähigkeit } \\
\text { deutlich langsamer }\end{array}$ \\
$\begin{array}{l}\text { Einfluss von Parteien- } \\
\text { differenzen }\end{array}$ & $\begin{array}{l}\text { Sinkt im Zeitalter per- } \\
\text { manenter Austerität, } \\
\text { steigt im Überschuss } \\
\text { wieder an }\end{array}$ & $\begin{array}{l}\text { Sinkt im Zeitalter perma- } \\
\text { nenter Austerität, bleibt } \\
\text { im Überschuss gering }\end{array}$ \\
\hline $\begin{array}{l}\text { Prognose für die Poli- } \\
\text { tik im Überschuss }\end{array}$ & $\begin{array}{l}\text { Politikwechsel gegen- } \\
\text { rung der Konsolidie- }\end{array}$ & $\begin{array}{l}\text { Fortsetzung der Konsoli- } \\
\text { dierungspolitik }\end{array}$ \\
\hline
\end{tabular}

Während im Zentrum der progressiven Konsolidierungsthese die Prognose eines Politikwechsels nach Ausgleich des Haushaltes steht, geht die Pfadabhängigkeitsthese von einer Fortsetzung der wichtigsten Trends der Konsolidierungspolitik aus. Auf Basis dieser Überlegungen lassen sich konkrete konkurrierende Thesen hinsichtlich der fiskalpolitischen Wirkung von Konsolidierungen und ihren Effekten auf die staatliche Handlungsfähigkeit formulieren, die in Tabelle 1 überblicksartig dargestellt werden. Zwar stimmen beide Ansätze überein, dass hohe Staatsschulden und Haushaltsdefizite die Gestaltungsfähigkeit eines Staates beschneiden und dass der fiskalische Handlungsspielraum durch eine erfolgreiche Haushaltskonsolidierung wieder erhöht werden kann. Allerdings kommen sie zu unterschiedlichen Schlussfolgerungen hinsichtlich der ,politischen Kollateralschäden“, die im Zuge der Haushaltssanierung entstehen. Deshalb prophezeit die 
Pfadabhängigkeitsthese, dass die Politikfelder, die in der Konsolidierung von besonders starken Einschnitten betroffen waren, auch besonders wenig von den neu gewonnenen Spielräumen profitieren.

Diese unterschiedlichen Annahmen über die Entwicklung des politischen Handlungsspielraums haben außerdem erhebliche Auswirkungen auf die Möglichkeiten der Umsetzung parteipolitischer Ziele in Überschusszeiten. Die progressive Konsolidierungsthese geht davon aus, dass Parteieneffekte im Überschuss zunehmen, weil die Parteien den gewonnenen fiskalischen Handlungsspielraum gemäß ihrer Präferenzen verwenden können. Dagegen sieht die Pfadabhängigkeitsthese kaum Chancen für wirksame Parteiendifferenzen, da die Zwänge des durch die Konsolidierung veränderten fiskalpolitischen Kontextes anhalten und sich die Parteien jeder politischen Färbung nach ihnen richten müssen.

Theoretisch sind Phänomene wie Pfadabhängigkeiten, Policy Feedback oder politische Erblasten, auf denen die Pfadabhängigkeitsthese beruht, vor allem in Ansätzen des historischen Institutionalismus beschrieben und konzeptualisiert worden. ${ }^{9}$ Die Folgewirkungen politischer Entscheidungen und Reformmaßnahmen sind im Kontext einer nachhaltigen Sanierung des Staatshaushalts besonders relevant. Schließlich ist es das erklärte Ziel der progressiven Konsolidierung, die Haushaltspolitik und ihre institutionellen Rahmenbedingungen tiefgreifend zu verändern, sodass sie den fiskalischen Handlungsspielraum eines Staates dauerhaft erhöht.

Konkret können nachhaltige Konsolidierungen mit Paul Pierson auf den drei Ebenen ,political interests, institutions and policy arrangements" politische Pfadabhängigkeiten auslösen. ${ }^{10}$ Besonders offenkundig sind die Einschränkungen des politischen Handlungsspielraums, die von institutionellen Reformen ausgehen. Fast immer werden im Zuge umfassender Haushaltskonsolidierungen institutionelle Reformen beschlossen, die zur Durchsetzung einer restriktiveren Fiskalpolitik beitragen sollen. ${ }^{11}$ Dabei kann es sich um numerische Überschussziele oder Schuldenobergrenzen handeln, ebenso wie um Maßnahmen für eine größere Transparenz des Budgetprozesses oder für seine Zentralisierung. Über derartige

9 Für Überblicksdarstellungen siehe zum Beispiel: Thelen, K.A.: Historical Institutionalism in Comparative Politics, in: Annual Review of Political Science, 2 (1999), 369-404; Pierson, P./Skocpol, T.: Historical Institutionalism in Contemporary Political Science, in: Katznelson, I./Milner, H.V. (Hg.): Political Science: State of the Discipline, New York, 2002, 693-721.

10 Pierson, P.: From Expansion to Austerity, in: Levin, M.A./Landy, M.K./Shapiro, M. (Hg.): Seeking the Center, Washington, DC, 2001, 54-80, hier 56f.

11 Diese werden in der Fachliteratur regelmäßig empfohlen: Von Hagen, J.: Budgeting Procedures and Fiscal Performance in the European Communities, Economic Paper 96, Brüssel, 1992. 
Instrumente noch hinaus geht die Einführung expliziter Ausgabengrenzen, die vorab festlegen, wie viel Geld in zukünftigen Jahren ausgegeben werden kann. ${ }^{12}$ Solche Grenzen stellen sicher, dass unerwartet gestiegene Einnahmen nicht in höhere Ausgaben fließen, sondern für den Schuldenabbau oder für Steuersenkungen eingesetzt werden. ${ }^{13}$ Aus der Perspektive der Pfadabhängigkeitsthese liegt die Bedeutung derartiger Reformen vor allem darin, dass sie wirksam bleiben, wenn der Konsolidierungserfolg eingetreten ist. Statt der Politik die Hände zu führen, dienen sie nun dazu, ihr die Hände zu binden.

Konsolidierungen gehen darüber hinaus zwangsläufig mit einer Neudefinition der fiskalpolitischen Prioritäten einher: Vor allem auf der Ausgabenseite des Budgets gehen der Erzielung von Haushaltsüberschüssen typischerweise umfangreiche Kürzungen voraus. ${ }^{14}$ Auch hier ist jedoch unwahrscheinlich, dass solche Maßnahmen nach dem Ende der Konsolidierung zurückgenommen werden, da ihre Durchsetzung in der Regel mit hohen politischen Kosten verbunden war. Sparmaßnahmen stoßen auf starken Widerstand in der Bevölkerung und erfordern daher eine umfassende politische Debatte. Diese bewirkt unter anderem einen Wandel des fiskalpolitischen Klimas, im Zuge dessen Gesetze und Reformen „fiskalisiert“ werden, ${ }^{15}$ was bedeutet, dass sie nicht mehr primär anhand fachpolitischer Vorund Nachteile, sondern vor allem in Bezug auf ihre budgetären Auswirkungen beurteilt werden. In einem solchen fiskalpolitischen Kontext stehen neue Gesetzesinitiativen von vornherein unter einem starken Finanzierungsvorbehalt.

Gleichzeitig ist ein ausgeglichener Haushalt jedoch auch ein großer politischer Erfolg, was sich unter anderem daran zeigt, dass Regierungen nach einer erfolgreichen Konsolidierung häufig wiedergewählt werden. ${ }^{16}$ In der Regel setzen die im Amt bestätigten Regierungen ihren restriktiven haushaltspolitischen Kurs fort, um ihre errungene Reputation für eine solide Haushaltsführung nicht wieder zu verlieren. Zugleich bemühen sich die Oppositionsparteien im politischen Wettstreit

12 Ljungman, G.: Expenditure Ceilings-A Survey, IMF Working Paper 08/282, 2008.

13 Zudem nehmen sie Zinskosten in der Regel von der Berechnung der Ausgabengrenze aus, sodass Zinsersparnisse nicht für neue Investitionen verwendet werden können: Ljungman, G.: a.a.O., hier: 8.

14 Nachhaltig erfolgreiche Konsolidierungen wurden typischerweise durch umfangreiche Ausgabenkürzungen und nicht durch Einnahmeerhöhungen erreicht: Alesina, A./Ardagna, S.: a.a.O.

15 White, J./Wildavsky, A.: The Deficit and the Public Interest: The Search for Responsible Budgeting in the 1980s, Berkeley, 1991; Lewis, T., a.a.O.

16 Brender, A./Drazen, A.: How do Budget Deficits and Economic Growth Affect Reelection Prospects? Evidence from a Large Panel of Countries, in: American Economic Review 98/5 (2008), 2203-2220; Alesina, A./Carloni, D./Lecce, G.: The Electoral Consequences of Large Fiscal Adjustments, in: Alesina, A./Giavazzi, F. (Hg.): Fiscal Policy after the Great Recession, Chicago, 2012, 531-570. 
als mindestens ebenso verantwortungsvolle Hüter der Staatsfinanzen zu erscheinen. Von der Opposition droht der Regierung im Überschuss häufig nicht etwa Kritik für zu wenig, sondern für zu viel fiskalpolitischen Aktivismus. Insbesondere läuft sie Gefahr, als unverantwortlich oder verschwenderisch angegriffen zu werden, wenn sie ein Abrücken von der „schwarzen Null“ in Erwägung zieht. Schließlich hat diese einen hohen und leicht verständlich zu machenden Symbolwert. Sie erfüllt damit alle Voraussetzungen, um in der politischen Debatte gegen die Regierung eingesetzt zu werden. ${ }^{17}$

Die für die Haushaltssanierung notwendige Austeritätspolitik verändert jedoch nicht nur das fiskalpolitische Klima, sondern auch die Interessenlagen in der Bevölkerung, was ebenfalls zu einer dauerhaften Reduzierung politischer Handlungsspielräume beiträgt. Denn die Sparmaßnahmen stärken bestimmte Interessengruppen und Politikziele, während sie gleichzeitig andere schwächen. Dies geschieht etwa, wenn sich der Staat im Zuge der Sparpolitik aus zuvor bedeutenden Aktivitäten zurückzieht, zum Beispiel indem er ihre Bereitstellung privatisiert. Immer mehr relevante Güter und Dienstleistungen werden dann nicht mehr vom Staat, sondern über den Markt organisiert. Wenn die Bürger aber elementare Dienstleistungen wie Gesundheitsvorsorge oder Bildung in wachsendem Maße auf privaten Märkten erwerben, wird verfügbares Einkommen für sie immer wichtiger. Folglich gewinnen Steuersenkungen gegenüber höheren Staatsausgaben an Bedeutung. Ohnehin werden bisherige Nettoempfänger staatlicher Transfers durch die Sparmaßnahmen zu Nettozahlern, was diesen Effekt noch einmal verstärkt. Der Rückbau staatlicher Leistungen wird darüber hinaus das Gewicht derjenigen reduzieren, die an ihrer Erstellung beteiligt sind oder die von diesen Leistungen profitieren. Zum Beispiel werden Gewerkschaften des öffentlichen Dienstes an Einfluss verlieren, wenn die Größe des öffentlichen Sektors und die Zahl seiner Beschäftigten abnehmen. ${ }^{18}$

In der Erklärungslogik der Pfadabhängigkeitsthese bewirken Konsolidierungen Veränderungen der fiskalischen Institutionen, der politischen Interessen und der konkreten fiskalpolitischen Maßnahmen, die den politischen Handlungsspielraum

17 Weaver, R.K.: The Politics of Blame Avoidance, in: Journal of Public Policy 6/4 (1986), 371-398, hier 379; Campbell, J.L.: Ideas, Politics, and Public Policy, in: Annual Review of Sociology 28 (2002), 21 38 , hier 29.

18 Schwartz, H.: Small States in Big Trouble: State Reorganization in Australia, Denmark, New Zealand, and Sweden in the 1980s, in: World Politics 46/4 (1994), 527-555. 
langfristig beschränken. Dabei kann von einem Wechsel des fiskalischen Regimes $^{19}$ gesprochen werden, weil diese Entwicklungstrends nicht unabhängig voneinander sind, sondern in dieselbe Richtung weisen und sich gegenseitig verstärken: Neue Vorstellungen von guter Fiskalpolitik lösen institutionelle Reformen aus, diese verändern die Politikmaßnahmen, neue Politikmaßnahmen wirken auf die Struktur der politischen Interessen, veränderte Interessen beeinflussen das strategische Kalkül der Parteien, die an der Regierung wiederum neue institutionelle Reformen beschließen. In Kombination bewirken diese Prozesse eine permanente Aufrechterhaltung des Spardrucks und der Ausgabenkürzungen und verfestigen damit die Austerität über die eigentliche Haushaltskonsolidierung hinaus.

Folgt man dieser Argumentation, dann werden die Vertreter einer progressiven Konsolidierung zu Opfern ihres eigenen Erfolgs, da Schuldenabbau durch Senkung öffentlicher Ausgaben von einem temporären Mittel zu einem permanenten politischen Ziel wird. Zwar gelingt es ihnen, den fiskalischen Spielraum wieder auszubauen, jedoch um den Preis, ihn aufgrund von politisch-institutionellen Restriktionen nicht wie gewünscht nutzen zu können. Die veränderten Rahmenbedingungen im Zusammenhang mit der erfolgreichen Haushaltskonsolidierung beschränken die Möglichkeiten für einen progressiven Politikwechsel. Die angestrebte Ausweitung der gestaltenden Staatstätigkeit kann nicht verwirklicht werden.

\section{Fallauswahl: Haushaltsüberschüsse in der OECD}

Die Entwicklung der Staatstätigkeit in Überschusszeiten wird im Folgenden empirisch überprüft. Gegenstand der Analyse ist dabei die Fiskalpolitik entwickelter, wohlfahrtsstaatlicher Demokratien zwischen 1980 und 2009, also im Zeitalter permanenter Austerität $t^{20}$, in dem die fiskalischen Handlungsspielräume angesichts fortschreitenden demographischen Wandels, zunehmender Globalisierung und sinkender Wachstumsraten unter immer stärkeren Druck gerieten. Diese dreißig Jahre kommen dem in Ländervergleichsstudien immer problematischen Ziel kausaler Homogenität ${ }^{21}$ so nah, wie es bei einem solchen Untersuchungsgegenstand möglich ist. Gerade in diesem Kontext schien das Versprechen einer Wiedergewinnung fiskalpolitischer Handlungsfähigkeit zudem besonders attraktiv.

19 Pierson, P.: a.a.O.

20 Pierson, P.: a.a.O.

21 King, G./Keohane, R.O./Verba, S.: Designing Social Inquiry, Princeton, 1994; Kittel, B./Winner, H.: How Reliable is Pooled Analysis in Political Economy? in: European Journal of Political Research 44/2 (2005), 269-293. 
Im Zentrum der folgenden Analysen stehen dabei Länder mit dauerhaften Haushaltsüberschüssen. In diesen Ländern waren die Bedingungen für die Gültigkeit der progressiven Konsolidierungsthese besonders günstig. Nicht nur gelang es ihnen, ihre Haushalte auszugleichen und das Wachstum der Staatsverschuldung zu stoppen. Vielmehr führten sie die Verschuldung sogar deutlich zurück. Damit sank auch ihre Zinslast, wodurch sich neue fiskalische Spielräume eröffneten. Zudem erzeugen Überschüsse tatsächlich verfügbaren Cashflow, also Einnahmen, denen noch kein Verwendungszweck zugewiesen ist und die somit im Prinzip diskretionär für Steuersenkungen, Mehrausgaben oder Sondertilgungen eingesetzt werden können. Darüber hinaus sind Länder mit Überschüssen in viel geringerem Maße auf das Wohlwollen der Finanzmärkte angewiesen. Aus all diesen Gründen sollten sie gemäß der progressiven Konsolidierungsthese erheblich an Handlungsfähigkeit gewinnen. Sie stellen somit einen Most-likely Case $^{22}$ für deren Gültigkeit dar.

Hinzu kommt, dass es auch deutlich mehr derartige Fälle gibt, als es der übliche Fokus auf einen Deficit Bias entwickelter Demokratien vermuten ließe. Dabei betrachten wir, um Einmaleffekte und Überschüsse im Promillebereich auszuschließen, im Folgenden nur Überschüsse, die mindestens zwei Jahre andauern und deren kumuliertes Volumen mindestens ein Prozent des BIP beträgt. Dabei endet eine Überschussperiode, wenn ein Land ein Defizit ausweist, ohne innerhalb der nächsten zwei Jahre wieder in den Überschuss zurückzukehren. Für Neuseeland bilden die Jahre 1994-2008 daher trotz kleiner Defizite in den Jahren 1998 und 1999 eine geschlossene Überschussperiode, da das Land bereits im Jahr 2000 wieder einen Überschuss erwirtschaftete.

Auf Basis dieser Periodendefinition erlebten immerhin 13 der 19 verbleibenden „klassischen“ OECD-Länder (ohne Norwegen) mit mehr als einer Million Einwohnern zwischen 1980 und 2009 mindestens eine Überschussperiode, wobei die einzelnen Perioden zwischen zwei und 15 Jahren dauerten (vgl. Tab. 2). Insgesamt wiesen die OECD-Länder in 121 Länder-Jahren oder 21,2 \% der Zeit eine Überschussperiode aus. Diese Überschüsse verteilen sich jedoch sehr unausgewogen auf die einzelnen Länder. Insgesamt entfielen drei Viertel der Haushaltsüberschüsse auf nur sieben Länder - Australien, Dänemark, Finnland, Irland, Kanada, Neuseeland und Schweden.

22 Eckstein, H.: Case Study and Theory in Political Science, in: Greenstein, F./Polsby, N. (Hg.): The Handbook of Political Science: Strategies of Inquiry, Reading, 1975, 79-137; Collier, D./Seawright, J.: Glossary, in: Brady, H./Collier, D. (Hg.): Rethinking Social Inquiry: Diverse Tools, Shared Standards, Lanham, 2010, 313-359, hier 339. 
Tabelle 2: Überschussperioden in OECD-Ländern, 1980-2009

\begin{tabular}{l|c|c}
\hline Land & $\begin{array}{c}\text { Dauer der } \\
\text { Überschussperiode }\end{array}$ & $\begin{array}{c}\text { Zeitraum der } \\
\text { Überschussperiode }\end{array}$ \\
\hline Australien & 10 & $1998-2007$ \\
Dänemark & 4 & $1986-1989$ \\
Dänemark & 10 & $1999-2008$ \\
Finnland & 11 & $1980-1990$ \\
Finnland & 11 & $1998-2008$ \\
Großbritannien & 3 & $1999-2001$ \\
Irland & 11 & $1997-2007$ \\
Japan & 5 & $1988-1992$ \\
Kanada & 11 & $1997-2007$ \\
Neuseeland & 15 & $1994-2008$ \\
Niederlande & 2 & $1999-2000$ \\
Niederlande & 3 & $2006-2008$ \\
Schweden & 4 & $1987-1990$ \\
Schweden & 11 & $1998-2008$ \\
Schweiz & $4+$ & $2006-$ offen \\
Spanien & 3 & $2005-2007$ \\
USA & 3 & $1998-2000$ \\
\hline
\end{tabular}

Damit sind diese Länder für eine sichtbare Zweiteilung des Datensatzes verantwortlich. Von den 17 Überschussperioden dauerten neun Perioden zwischen zwei und fünf Jahren, die übrigen acht Perioden aber zehn Jahre oder mehr. Dagegen gab es keinen Überschuss, der zwischen sechs und neun Jahren dauerte. Diese bipolare Verteilung der Dauer der Überschüsse ist Ausdruck systematischer Unterschiede zwischen den beiden Gruppen, sowohl in Bezug auf die Entstehung als auch die Bewahrung der Überschüsse. ${ }^{23}$

Die kurzen Überschüsse ergaben sich immer dann, wenn ein Land auf dem Gipfel des Konjunkturzyklus dank steigender Einnahmen und fallender Ausgaben den Haushalt ausglich. Dieser Überschuss signalisierte jedoch nicht viel mehr als einen außergewöhnlich starken Wirtschaftsaufschwung. Es wurden kaum politische

23 Siehe dazu detailliert Haffert, L.: Freiheit von Schulden - Freiheit zur Gestaltung? Die politische Ökonomie von Haushaltsüberschüssen, Univ. Diss., Universität zu Köln und Max-Planck-Institut für Gesellschaftsforschung, 2014, hier 88f. 
Maßnahmen zu seiner Bewahrung getroffen und der Überschuss verschwand entsprechend schnell, wenn der Konjunkturaufschwung zu Ende ging. In den langen Perioden entkoppelten sich die Überschüsse dagegen weitgehend vom Konjunkturzyklus und wurden auch durch Abschwünge hindurch bewahrt.

Vor diesem Hintergrund konzentrieren sich die folgenden Analysen auf die Länder, die ihre Überschüsse für mehr als eine Dekade bewahren konnten. Wegen der Besonderheiten der finnischen politischen Ökonomie wird dabei jedoch der finnische Überschuss der 1980er-Jahre ausgeklammert, insbesondere weil er nicht das Ergebnis einer Konsolidierung, sondern die Fortsetzung einer permanenten Überschusspolitik war. ${ }^{24}$ Ebenfalls nicht berücksichtigt wird der Haushaltsüberschuss Irlands, weil dieser kein struktureller Überschuss, sondern Ausdruck einer Immobilienblase sowie einer allgemeinen Überhitzung der Wirtschaft war. Beide Überschussperioden sind insofern kaum für einen Vergleich geeignet.

Tabelle 3: Fiskalischer Handlungsspielraum in sechs Überschussländern, in Prozent des BIP

\begin{tabular}{|c|c|c|c|c|c|c|}
\hline \multirow[t]{2}{*}{ Land } & \multirow[t]{2}{*}{$\begin{array}{c}\text { Überschuss } \\
\text { seit }\end{array}$} & \multirow{2}{*}{$\begin{array}{c}\varnothing \\
\text { Über- } \\
\text { schuss }\end{array}$} & \multicolumn{2}{|c|}{$\begin{array}{l}\text { Nettoverschul- } \\
\text { dung }\end{array}$} & \multicolumn{2}{|c|}{ Nettozinslast } \\
\hline & & & in $t-1$ & 2007 & in $t-1$ & 2007 \\
\hline Australien & 1998 & 0,86 & 21,2 & $-7,3$ & 1,4 & 0,0 \\
\hline Dänemark & 1999 & 2,39 & 35,1 & $-3,8$ & 2,7 & 0,4 \\
\hline Finnland & 1998 & $3,48^{25}$ & $-7,5$ & $-72,6$ & 1,8 & $-0,6$ \\
\hline Kanada & 1997 & 0,98 & 68,5 & 22,4 & 5,2 & 0,6 \\
\hline Neuseeland & 1994 & 2,68 & 50,5 & $-5,5$ & 3,2 & 0,1 \\
\hline Schweden & 1998 & 1,23 & 24,6 & $-22,4$ & 3,0 & 0,7 \\
\hline Durchschnitt & / & 1,94 & 32,1 & $-14,9$ & 2,9 & 0,2 \\
\hline
\end{tabular}

Quelle: OECD Economic Outlook Database, eigene Berechnungen

Die sechs verbleibenden Fälle erfüllen dagegen die Bedingungen für eine Überprüfung der progressiven Konsolidierungsthese in besonderer Weise. Wie Tabelle 3 zeigt, verfügten sie tatsächlich über erheblichen und dauerhaften neuen fiskalischen Handlungsspielraum in Form von Überschusseinnahmen und sinkenden

24 Vartiainen, J.: The Finnish Model of Economic and Social Policy - from Cold War Primitive Accumulation to Generational Conflicts? in: Mjoset, L. (Hg.): The Nordic Varieties of Capitalism, Bingley, 2011, 53-87.

25 Finnlands extrem hohe Überschüsse erklären sich aus einer Besonderheit seines Rentensystems, das eine kapitalgedeckte Säule enthält, die über das staatliche Versicherungssystem organisiert wird und jährliche Rücklagen bildet, die in den 1990er-Jahren zeitweilig mehr als $3 \%$ des BIP erreichten. Die OECD rechnet diese Rücklagen dem Budgetsaldo des Gesamtstaates zu. 
Zinslasten. Dies war bei den kurzen Überschussepisoden dagegen nicht der Fall. Statt neue fiskalische Spielräume nutzen zu können, musste sich die Politik dort sehr bald wieder dem Ziel der Defizitbekämpfung zuwenden, weil der Konsolidierungserfolg nicht bewahrt wurde. Auch konnten die sechs untersuchten Länder zunehmend unabhängig vom Druck der Finanzmärkte agieren, was unter anderem daran deutlich wird, dass sie nach einigen Jahren im Überschuss ihre AAA-Ratings zurückerhielten, die sie im Vorfeld der Konsolidierung verloren hatten. ${ }^{26}$

Vertreter der progressiven Konsolidierungsthese könnten einwenden, die Beschränkung der Fallauswahl auf sechs Länder mit dauerhaften Überschüssen führe zu einer Verzerrung der Ergebnisse, weil sich diese Länder durch eine restriktive und gerade nicht gestaltende Fiskalpolitik selbst in den Datensatz hineinselektierten. Länder, die ihre Gestaltungsausgaben erhöhten, hätten ihren Überschuss dagegen wieder verloren und seien deshalb nicht im Datensatz. Diesem Argument ist aus Sicht der Pfadabhängigkeitsthese auch vollkommen zuzustimmen, argumentiert diese doch gerade, dass die dauerhafte Bewahrung von Überschüssen Maßnahmen erfordert, die gleichzeitig ihre Verwendung beschränken. Länder, die einen Politikwechsel gerade durch Verzicht auf eine Bewahrung des Überschusses erreichten, sind mit diesem Argument also vollkommen kompatibel. Um diesen Punkt noch weiter auszuführen, werden die folgenden Analysen daher auch einen kurzen Blick auf die Entwicklung in den übrigen Überschussländern werfen.

\section{Empirische Evidenz}

Die folgende empirische Analyse hat das Ziel zu untersuchen, wie die sechs Überschussländer ihre neu gewonnenen fiskalischen Spielräume verwendet haben. Dabei gilt es eine Besonderheit zu beachten: Überschüsse bilden die Differenz aus Einnahmen und Ausgaben und verschwinden, sobald sie auf einer der beiden Seiten des Budgets verwendet werden. Ihre Verwendung kann deshalb nicht unmittelbar beobachtet werden und wird als die Veränderung der Staatsausgaben und Staatseinnahmen in einem Haushaltsjahr operationalisiert, das auf einen Überschuss folgt.

Konkret leistet die folgende Analyse zweierlei: Zunächst wird die Entwicklung verschiedener aggregierter Maße der Staatstätigkeit in Konsolidierung und Überschuss deskriptiv in einer Längsschnittperspektive untersucht, um zu überprüfen, 
ob es zu dem von der progressiven Konsolidierungsthese prophezeiten Politikwechsel kam, oder ob sich die Trends der Konsolidierung auch im Überschuss fortsetzten. Danach wird die Entwicklung der verschiedenen Dimensionen fiskalpolitischer Handlungsfähigkeit mithilfe einer multivariaten Regression überprüft. Diese ergänzt eine Querschnittsperspektive und wirft die Frage auf, ob es den Überschussländern gelang, signifikant mehr Ressourcen für Gestaltungsaufgaben zu mobilisieren, als Ländern mit Defiziten.

Abbildung 1: Entwicklung von Staatsausgaben und Staatseinnahmen in sechs Überschussländern ${ }^{27}$

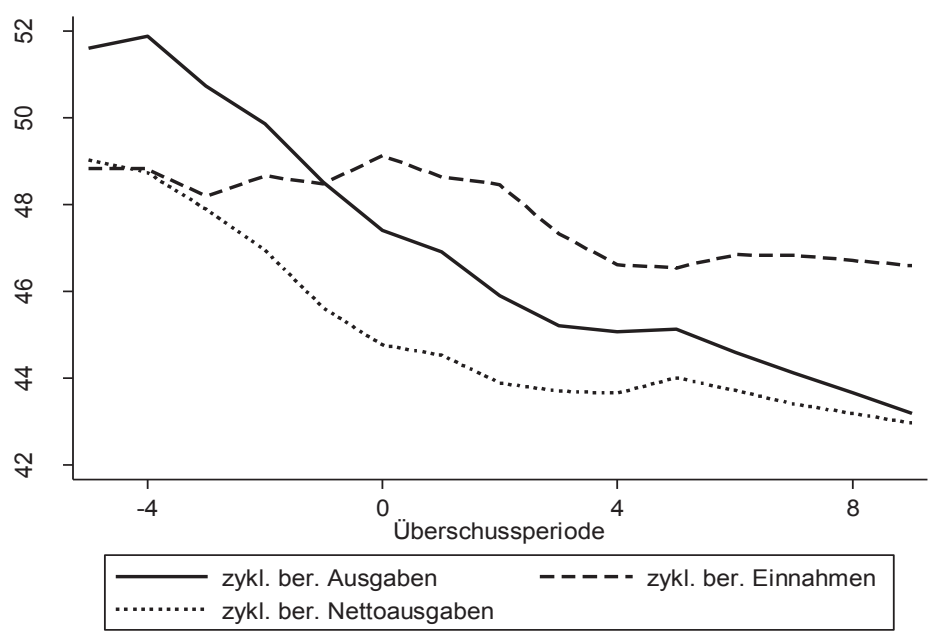

Quelle: OECD Economic Outlook Database, eigene Berechnungen

Die am stärksten aggregierte Perspektive auf den Staatshaushalt bietet die simple Betrachtung von Einnahmen und Ausgaben (vgl. Abb. 1). Dabei zeigt sich, dass die Überschüsse fast vollständig einer Kürzung der Staatsausgaben zu verdanken waren. ${ }^{28}$ Während die zyklisch bereinigten Einnahmen in der Konsolidierungsphase praktisch konstant blieben, sanken die zyklisch bereinigten Ausgaben um etwa vier Prozent des BIP. Die Entstehung der Überschüsse erfolgte also auf der Ausgabenseite. Dagegen erfolgte die Verwendung auf der Einnahmenseite: Die

27 In dieser Darstellung ist das Jahr “-1" das letzte Konsolidierungsjahr, das Jahr "0" das erste Überschussjahr.

28 Diesen Befund bestätigen auch maßnahmenbasierte Analysen: DeVries, P./Guajardo, J./Leigh, D./Pescatori, A.: A New Action-based Dataset of Fiscal Consolidation, IMF Working Paper 11/128, 2011. 
zyklisch bereinigten Einnahmen begannen im Überschuss nicht etwa wieder zu steigen, sondern gingen sogar weiter zurück. Das gilt sogar, wenn man Zinsausgaben ausschließt und die Entwicklung der Nettoausgaben betrachtet. Von den freiwerdenden Mitteln, die bislang durch Zinsausgaben gebunden waren, profitierte fast ausschließlich die Einnahmenseite des Budgets.

Abbildung 2: Entwicklung der Sozial- und der Nettokernausgaben als Anteil der gesamten Nettoausgaben in sechs Überschussländern

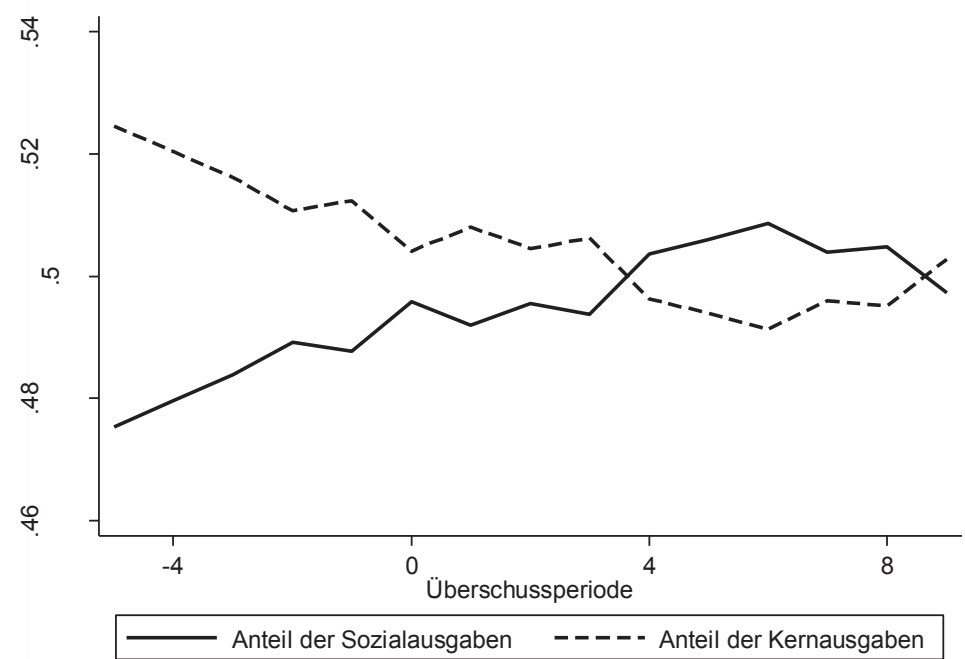

Quelle: OECD Economic Outlook Database, eigene Berechnungen

Blickt man auf die einzelnen Bestandteile des Budgets, so bietet es sich zunächst an, zwischen den Sozialausgaben und allen übrigen Ausgaben (ohne Zinsen), den sogenannten Nettokernausgaben, zu unterscheiden. ${ }^{29}$ Abbildung 2 zeigt die relativen Anteile der Kern- und der Sozialausgaben an den gesamten Nettoausgaben im Zeitverlauf. Dabei wird deutlich, dass die Kernausgaben den größeren Teil der Konsolidierungslast zu tragen hatten. Dagegen erwies sich der Wohlfahrtsstaat, in Übereinstimmung mit der Literatur, als verhältnismäßig geschützt. ${ }^{30}$ Zudem setzten sich die Trends der Konsolidierung auch hier im Überschuss weiter fort: So

29 Castles, F.G.: Testing the Retrenchment Hypothesis: An Aggregate Overview, in: Castles, F.G. (Hg.): The Disappearing State? Retrenchment Realities in an Age of Globalisation, Cheltenham, 2007, 19-43.

30 Breunig, C./Busemeyer, M., a.a.O. 
begann der Anteil der Nettokernausgaben nicht wieder zu steigen, sondern ging sogar weiter zurück.

Das spezifischste Maß staatlicher Gestaltungstätigkeit sind schließlich die öffentlichen Investitionen. Diese sind gemäß der ökonomischen neuen Wachstumstheorie $^{31}$ eine wichtige Quelle von Wirtschaftswachstum. Der klassische Indikator für die staatliche Investitionstätigkeit sind die harten Infrastrukturinvestitionen, definiert als Gross Fixed Capital Formation..$^{32}$ Allerdings wird zunehmend angeführt, dass Sachkapitalinvestitionen in einer wissensbasierten Dienstleistungsökonomie kein angemessenes Maß der staatlichen Gestaltungsausgaben mehr darstellen. Streeck und Mertens schlagen daher einen Indikator für Soft Public Investment vor, der aus den Ausgaben für Bildung, Forschung \& Entwicklung, Familienpolitik und aktive Arbeitsmarktpolitik zusammengesetzt ist. ${ }^{33}$ Diese weichen Investitionen sind auch ein Kernelement beim Aufbau eines vorsorgenden Sozialstaats oder Social Investment Welfare State. ${ }^{34}$

Die Entwicklung dieser beiden Formen öffentlicher Investitionen zeigt Abbildung 3. Dabei wurden sowohl die harten als auch die weichen Investitionen während der Konsolidierung erheblich gekürzt. Die weichen Investitionen erholten sich zu Beginn der Überschussperiode zunächst leicht, begannen dann aber wieder zu fallen und lagen nach 10 Jahren im Überschuss niedriger als im ersten Überschussjahr. Bei den Infrastrukturinvestitionen ist dagegen ein gewisser Wiederanstieg feststellbar. Dieser blieb jedoch hinter den in der Konsolidierung erfolgten Einschnitten zurück. Folgerichtig lag der öffentliche Nettokapitalstock der Überschussländer im Jahr 2001 deutlich niedriger als noch im Jahr 1990. ${ }^{35}$

31 Romer, P.M.: Endogenous Technological Change, in: Journal of Political Economy, 98/5 (1990), 71-102; Aghion, P./Howitt, P.: A Model of Growth Through Creative Destruction, NBER Working Paper No. 3223, 1990.

32 Keman, H.: Cutting Back Public Investment after 1980: Collateral Damage, Policy Legacies and Political Adjustment, in: Journal of Public Policy, 30/2 (2010), 163-182; Breunig, C./Busemeyer, M., a.a.O.

33 Streeck, W./Mertens, D.: Fiscal Austerity and Public Investment. Is the Possible the Enemy of the Necessary? MPIfG Working Paper 11/12, 2011. Siehe auch: Nikolai, R.: Towards Social Investment? Patterns of Public Policy in the OECD World, in: Morel, N./Palier, B./Palme, J. (Hg.): Towards a Social Investment Welfare State? Ideas, Policies and Challenges, Bristol, 2012, 91-115.

34 Esping-Andersen, G./Gallie, D./Hemerijck, A./Myles, J.: Why We Need a New Welfare State, Oxford, 2002; Morel, N./Palier, B./Palme, J. (Hg.): a.a.O.

35 Kamps, C.: New Estimates of Government Net Capital Stocks for 22 OECD Countries, 1960-2001, IMF Staff Papers 53/1, 2006. 
Abbildung 3: Entwicklung der harten und weichen Investitionen in sechs Überschussländern

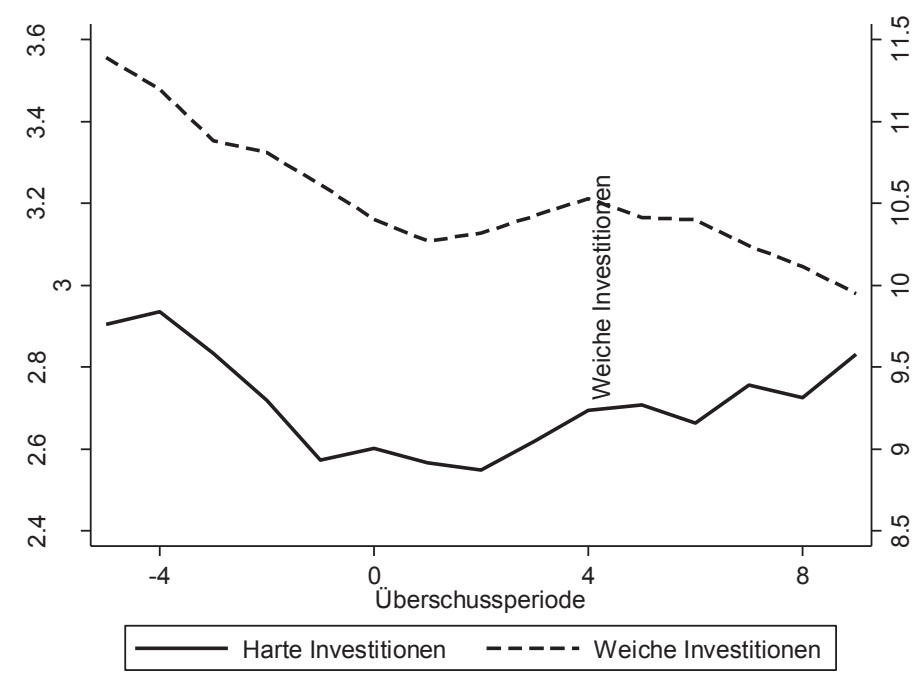

Quelle: OECD Economic Outlook Database, eigene Berechnungen

In keinem der bislang untersuchten Aggregate ist der von der progressiven Konsolidierungsthese erhoffte fiskalpolitische Kurswechsel feststellbar. Vielmehr setzten sich die Trends der Konsolidierung im Überschuss fort: Die Politikfelder, die besonders stark von der Konsolidierung betroffen waren, profitierten besonders wenig vom Überschuss. Dieses Ergebnis muss allerdings nicht zwangsläufig auf eine investitionsfeindliche Politik der Regierung zurückzuführen sein. Stattdessen könnten schlicht Faktoren wie Globalisierung, demographischer Wandel oder sinkende Wachstumsraten dafür verantwortlich sein. Möglicherweise wären die Ausgaben noch stärker gefallen, wenn die Länder keine Überschüsse erwirtschaftet hätten. Um diesen kontrafaktischen Überlegungen gerecht zu werden, ist es nötig, die Entwicklung der gestaltenden Staatstätigkeit mit der in anderen Ländern zu vergleichen und dabei den Einfluss solcher Faktoren zu kontrollieren.

Dies geschieht im Folgenden unter Verwendung eines Time-Series-Cross-Section (TSCS)-Designs für den gesamten Datensatz. Dabei wird zunächst geprüft, welcher Zusammenhang zwischen dem Haushaltssaldo und der Entwicklung der Staatstätigkeit ganz allgemein besteht. Danach wird untersucht, ob dieser Zusammenhang auch für den Fall von Überschüssen gilt. 
Im ersten Schritt wird folgende Regression für die verschiedenen Indikatoren der Entwicklung der Staatstätigkeit geschätzt (vgl. Tab. 5).

$\Delta$ Depvar $_{\mathrm{it}}=\alpha+\beta_{1} \times$ Depvar $_{\mathrm{it}-1}+\beta_{2} \times \Delta$ Depvar $_{\mathrm{it}-1}+\beta_{3} \times$ Saldo $_{\text {it- } 1}+\beta_{4} \times$ Kontrollvariablen $_{\mathrm{it}}+\varepsilon_{\mathrm{it}}$

Als abhängige Variablen werden dabei dieselben Maße wie in den Abbildungen 1-3 verwendet, also die zyklisch bereinigten Staatseinnahmen, die Nettokernausgaben, die Sozialausgaben sowie die harten und weichen Investitionen. Die in den Regressionen verwendeten Kontrollvariablen werden in Tabelle 4 vorgestellt.

Tabelle 4: In den Regressionen verwendete Variablen ${ }^{36}$

\begin{tabular}{l|l}
\hline Variable & Definition \\
\hline Saldo & Haushaltssaldo in Prozent des BIP \\
\hline Wachstum & Wachstumsrate des BIP in Prozent \\
\hline Arbeitslosigkeit & Arbeitslosenquote nach OECD-Definition \\
\hline Handel & $\begin{array}{l}\text { Außenhandel (Summe aus Importen und Exporten) in } \\
\text { Prozent des BIP }\end{array}$ \\
\hline Kapitalverkehr & Index für den Grad der Kapitalverkehrsfreiheit \\
\hline Log BIP pro Kopf & BIP pro Kopf in US-Dollar, logarithmiert \\
\hline Realzins & $\begin{array}{l}\text { Langfristiger Realzinssatz auf Staatsanleihen in Pro- } \\
\text { zent }\end{array}$ \\
\hline $\begin{array}{l}\text { Progressive Regie- } \\
\text { rung }\end{array}$ & Kabinettsanteil progressiver Parteien \\
\hline Junge & $\begin{array}{l}\text { Anteil der Personen unter 25 Jahren an der Gesamtbe- } \\
\text { völkerung }\end{array}$ \\
\hline Jahr & Kalenderjahr \\
\hline
\end{tabular}

Da es bei dem Ziel der Wiedergewinnung staatlicher Handlungsfähigkeit um deren $\mathrm{Zu}$ - oder Abnahme geht, verwendet die Regression Veränderungsraten als abhängige Variablen. Zudem enthält sie einen Zeittrend, um den allgemeinen Trend zu immer stärkerer Austerität in den OECD-Ländern abzubilden. ${ }^{38}$ Dagegen enthält

36 Die Quelle für die meisten Variablen ist die OECD Economic Outlook Database No. 92. Die Bildungsausgaben werden ergänzt mit Daten von Busemeyer (persönliche Kommunikation). Daten zur Parteienherrschaft und zur Kapitalverkehrsfreiheit stammen aus Armingeon, K./Weisstanner, D./Engler, S./Potolidis, P./Gerber, M.: Comparative Political Data Set I 1960-2010, Bern, 2012.

37 Anders als Armingeon et al., a.a.O. verwenden wir jedoch ein binäres Maß der politischen Lager. Dabei klassifizieren wir die kanadischen Liberalen und die US-Demokraten als progressive Parteien, während wir die christdemokratischen Parteien des europäischen Kontinents dem konservativen Lager zuordnen.

38 Fixe Zeiteffekte oder Dekadendummys anstelle eines Zeittrends zu verwenden, verändert die Ergebnisse qualitativ kaum. Auch die Aufnahme fixer Ländereffekte beeinflusst nur deren Signifikanz, wenngleich deutlich stärker, was jedoch aus den angeführten theoretischen Gründen zu erwarten ist; siehe Tab. Al im Anhang. 
die Regression in Übereinstimmung mit Plümper et al. ${ }^{39}$ keine fixen Ländereffekte. Der Grund dafür ist, dass der fixe Effekt die unabhängigen Variablen um ihren durchschnittlichen Wert innerhalb eines Landes bereinigt. Damit würden alle Niveauunterschiede zwischen Ländern aus der Regression entfernt. Die Pfadabhängigkeitsthese unterstellt aber gerade einen solchen Niveaueffekt in Form eines eigenen, zusätzlichen Effekts der Existenz von Überschüssen. Es widerspräche daher dem Zweck der Analyse, diesen Effekt in einen Dummy auszulagern.

Diese Regression stellt nur einen Zwischenschritt bei der Überprüfung der progressiven Konsolidierungsthese dar. Ihr Ziel ist weder eine vollständige Analyse der Staatstätigkeit noch eine Untersuchung der Signifikanz einzelner Kontrollvariablen, mit Ausnahme der Parteiendifferenz. Bei dieser zeigt sich weder ein direkter Effekt progressiver Regierungen auf die Entwicklung der Staatstätigkeit, noch ein indirekter Effekt, der über die Interaktion mit dem Haushaltssaldo vermittelt wäre. Das Regressionsergebnis bestätigt insofern die These, dass Parteieneffekte im Zeitalter permanenter Austerität an Bedeutung verloren haben, weil die Fiskalpolitik zunehmend von Sachzwängen geprägt wird.

Der Hauptzweck der Regression ist zu zeigen, dass der Budgetsaldo, bezogen auf den gesamten Datensatz, tatsächlich in allen Variablen den von der progressiven Konsolidierungsthese vorhergesagten Effekt hat: Die Veränderung der gestaltenden Staatstätigkeit variiert signifikant mit dem Budgetsaldo der Vorperiode. Demnach sorgt ein Defizit von $1 \%$ des BIP für einen Rückgang der Nettokernausgaben um $0,142 \%$ des BIP, für einen Rückgang der Sozialausgaben um 0,023\%, sowie für Kürzungen der Infrastrukturinvestitionen um 0,016 \% des BIP und der weichen Investitionen um 0,034 \% des BIP. Zugleich ist es mit einer Erhöhung der Einnahmen um 0,062 \% des BIP verbunden. Auch hier gilt, dass der Konsolidierungsdruck wesentlich stärkere Reaktionen auf der Ausgaben- als auf der Einnahmenseite auslöst.

39 Plümper, T./Manow, P./Troeger, V.: Panel Data Analysis in Comparative Politics: Linking Method to Theory, in: European Journal of Political Research, 44/2 (2005), 327-354. 
Tabelle 5: Regression für die Entwicklung der Staatstätigkeit in 19 OECD-Ländern, 1980-2009

\begin{tabular}{|c|c|c|c|c|c|}
\hline & $\begin{array}{c}\text { Netto- } \\
\text { kern }\end{array}$ & $\begin{array}{c}\text { Sozial- } \\
\text { staat }\end{array}$ & Weich & Hart & $\begin{array}{c}\text { Einnah- } \\
\text { men }\end{array}$ \\
\hline Saldo (t-1) & $\begin{array}{c}0.142 * * * \\
(0.022)\end{array}$ & $\begin{array}{c}0.023 * * \\
(0.008)\end{array}$ & $\begin{array}{c}0.034 * * * \\
(0.008)\end{array}$ & $\begin{array}{c}0.016 * * \\
(0.005)\end{array}$ & $\begin{array}{c}-0.062 * * * \\
(0.018)\end{array}$ \\
\hline Wachstum (t-1) & $\begin{array}{l}-0.162 * \\
(0.074)\end{array}$ & $\begin{array}{c}-0.184 * * * \\
(0.020)\end{array}$ & $\begin{array}{c}- \\
0.082^{* * *} \\
(0.016)\end{array}$ & $\begin{array}{c}0.008 \\
(0.013)\end{array}$ & $\begin{array}{c}0.037 \\
(0.035)\end{array}$ \\
\hline$\Delta$ Wachstum & $\begin{array}{c}-0.163 * * \\
(0.051)\end{array}$ & $\begin{array}{c}-0.265^{* * *} \\
(0.016)\end{array}$ & $\begin{array}{c}- \\
0.105^{* * *} \\
(0.013)\end{array}$ & $\begin{array}{l}-0.012 \\
(0.009)\end{array}$ & \\
\hline$\Delta$ Arbeitslosigkeit & $\begin{array}{l}0.203 * \\
(0.090)\end{array}$ & $\begin{array}{c}0.085 * * \\
(0.032)\end{array}$ & $\begin{array}{l}-0.001 \\
(0.023)\end{array}$ & $\begin{array}{c}0.012 \\
(0.019)\end{array}$ & $\begin{array}{c}-0.211 * * \\
(0.066)\end{array}$ \\
\hline Handel & $\begin{array}{l}-0.001 \\
(0.001)\end{array}$ & $\begin{array}{l}-0.001 \\
(0.001)\end{array}$ & $\begin{array}{c}0.000 \\
(0.001)\end{array}$ & $\begin{array}{c}- \\
0.001^{* * *} \\
(0.000)\end{array}$ & $\begin{array}{c}0.000 \\
(0.001)\end{array}$ \\
\hline Kapitalverkehr & $\begin{array}{c}-0.250 * * \\
(0.093)\end{array}$ & $\begin{array}{l}-0.019 \\
(0.030)\end{array}$ & $\begin{array}{c}0.015 \\
(0.030)\end{array}$ & $\begin{array}{l}-0.023 \\
(0.017)\end{array}$ & $\begin{array}{l}-0.092 \\
(0.064)\end{array}$ \\
\hline Log BIP pro Kopf & $\begin{array}{c}0.462 \\
(0.320)\end{array}$ & $\begin{array}{l}-0.118 \\
(0.119)\end{array}$ & $\begin{array}{l}-0.139 \\
(0.117)\end{array}$ & $\begin{array}{l}-0.015 \\
(0.083)\end{array}$ & $\begin{array}{c}0.096 \\
(0.269)\end{array}$ \\
\hline Realzins & $\begin{array}{c}0.058 \\
(0.046)\end{array}$ & $\begin{array}{c}0.053 * * * \\
(0.015)\end{array}$ & $\begin{array}{l}0.032 * \\
(0.015)\end{array}$ & $\begin{array}{c}0.022 * * \\
(0.008)\end{array}$ & $\begin{array}{c}0.028 \\
(0.036)\end{array}$ \\
\hline progressive Regierung & $\begin{array}{l}-0.121 \\
(0.152)\end{array}$ & $\begin{array}{c}0.072 \\
(0.058)\end{array}$ & $\begin{array}{c}0.048 \\
(0.050)\end{array}$ & $\begin{array}{c}0.024 \\
(0.031)\end{array}$ & $\begin{array}{l}-0.022 \\
(0.112)\end{array}$ \\
\hline Progressiv*Saldo & $\begin{array}{c}0.030 \\
(0.028)\end{array}$ & $\begin{array}{c}0.007 \\
(0.014)\end{array}$ & $\begin{array}{l}-0.004 \\
(0.012)\end{array}$ & $\begin{array}{c}0.012 \\
(0.007)\end{array}$ & $\begin{array}{l}-0.019 \\
(0.027)\end{array}$ \\
\hline Jahr & $\begin{array}{l}-0.020 \\
(0.016)\end{array}$ & $\begin{array}{c}0.012 \\
(0.007)\end{array}$ & $\begin{array}{c}0.004 \\
(0.005)\end{array}$ & $\begin{array}{c}0.005 \\
(0.004)\end{array}$ & $\begin{array}{l}-0.003 \\
(0.016)\end{array}$ \\
\hline$\Delta$ Nettokern $(\mathrm{t}-1)$ & $\begin{array}{c}- \\
0.281 * * * \\
(0.069)\end{array}$ & & & & \\
\hline Nettokern (t-1) & $\begin{array}{c}- \\
0.069^{* * *} \\
(0.019)\end{array}$ & & & & \\
\hline$\Delta$ Sozialausgaben $(\mathrm{t}-1)$ & & $\begin{array}{c}0.248 * * * \\
(0.042)\end{array}$ & & & \\
\hline Sozialausgaben (t-1) & & $-0.027 * * *$ & & & \\
\hline
\end{tabular}




\begin{tabular}{|c|c|c|c|c|c|}
\hline & $\begin{array}{l}\text { Netto- } \\
\text { kern }\end{array}$ & $\begin{array}{c}\text { Sozial- } \\
\text { staat }\end{array}$ & Weich & Hart & $\begin{array}{c}\text { Einnah- } \\
\text { men }\end{array}$ \\
\hline \multirow{3}{*}{$\Delta$ Weiche Investitionen $(\mathrm{t}-1)$} & & $(0.005)$ & & & \\
\hline & & & 0.071 & & \\
\hline & & & $(0.053)$ & & \\
\hline \multirow[t]{2}{*}{ Weiche Investitionen ( $\mathrm{t}-1)$} & & & $\begin{array}{c}- \\
0.050 * * *\end{array}$ & & \\
\hline & & & $(0.011)$ & & \\
\hline \multirow[t]{2}{*}{ Delta Junge } & & & 0.056 & & \\
\hline & & & $(0.132)$ & & \\
\hline \multirow[t]{2}{*}{$\Delta$ Harte Investitionen $(\mathrm{t}-1)$} & & & & 0.031 & \\
\hline & & & & $(0.071)$ & \\
\hline \multirow[t]{2}{*}{ Harte Investitionen (t-1) } & & & & $-0.053 * *$ & \\
\hline & & & & $(0.017)$ & \\
\hline \multirow[t]{2}{*}{$\Delta$ zykl. ber. Einnahmen (t-1) } & & & & & $0.128^{*}$ \\
\hline & & & & & $(0.058)$ \\
\hline \multirow[t]{2}{*}{ zykl. ber. Einnahmen (t-1) } & & & & & -0.011 \\
\hline & & & & & $(0.007)$ \\
\hline \multirow[t]{2}{*}{ Konstante } & 38.678 & -20.917 & -5.120 & -9.211 & 4.903 \\
\hline & -30.018 & -13.518 & -9.664 & -8.349 & -31.313 \\
\hline $\mathrm{N}$ & 468 & 484 & 359 & 495 & 485 \\
\hline $\mathrm{r} 2$ & 0.293 & 0.583 & 0.332 & 0.160 & 0.179 \\
\hline
\end{tabular}

Panelkorrigierte Standardfehler in Klammern, ${ }^{*} \mathrm{p}<0.05, * * \mathrm{p}<0.01, * * * \mathrm{p}<0.001$

Die für diese Studie entscheidende Frage lautet, ob diese Zusammenhänge auch im Kontext von dauerhaften Überschüssen gelten. Dabei prognostiziert die progressive Konsolidierungsthese eine symmetrische Entwicklung, wonach die Ausgaben in den Politikfeldern, in denen besonders stark gekürzt wurde, danach ceteris paribus wieder besonders deutlich steigen. Dagegen impliziert die Pfadabhängigkeitsthese, dass diese Symmetrieannahme nicht zutrifft. Verwendet man die Regressionsergebnisse, um die Entwicklung der Staatstätigkeit im Überschuss vorherzusagen, sollte dies zu signifikant zu optimistischen Prognosen für diese besonders betroffenen Bereiche führen.

Diese widerstreitenden Hypothesen können mithilfe einer Analyse der Residuen der Regression überprüft werden. Qua Konstruktion des Regressionsmodells ist 
der Mittelwert der Residuen für den gesamten Datensatz Null. Auch sollte die Spezifikation des Modells in Veränderungsraten dafür gesorgt haben, dass keine Autokorrelation in den Residuen vorhanden ist, zumal die Modelle auch für die Veränderungsrate der Vorperiode kontrollieren. Tatsächlich bestätigen einfache Autokorrelations-Tests, dass die Residuen der Vorperiode, bezogen auf den gesamten Datensatz, keinen Effekt auf die aktuellen Residuen haben. Das muss aber nicht für alle Subgruppen des Datensatzes gelten. Es ist durchaus möglich, dass die Regression für einen bestimmten Teil des Datensatzes systematisch zu optimistische Prognosen oder zu pessimistische Prognosen trifft. Konkret lässt sich fragen, wie gut die Regression die Entwicklung der Staatstätigkeit in den 68 Überschussjahren prognostiziert.

Abbildung 4 zeigt deshalb die tatsächlichen jährlichen Zuwachsraten der Staatsausgaben in den 68 Überschussjahren im Verhältnis zu den von der Regression prognostizierten Werten. Dabei liegt der Schwerpunkt der Punktewolken in allen vier Kategorien sichtbar unter der Geraden, auf der prognostizierte und realisierte Werte einander entsprechen. Besonders deutlich tritt dieser Effekt bei den Nettokernausgaben hervor, wo die realisierten Werte im Durchschnitt um erstaunliche $0,37 \%$ des BIP (p-Wert: 0,000 ) unter den prognostizierten Werten liegen. Weniger eindrucksvoll, aber immer noch annähernd signifikant ist dieser Effekt bei den weichen Investitionen, wo die Abweichung $0,07 \%$ des BIP beträgt (p-Wert: 0,051). Bei den harten Investitionen schließlich beträgt die Abweichung 0,04 \% des BIP (p-Wert: 0,036). Von dieser Zurückhaltung bei den Investitionen profitiert jedoch nicht etwa der Wohlfahrtsstaat. Vielmehr bleibt dieser im Überschuss um $0,13 \%$ des BIP hinter den Prognosen zurück (p-Wert 0,016). ${ }^{40}$ die Überschussjahre bereits in die Ausgangsregression einfließen und den geschätzten Effekt des Haushaltssaldos dort bereits reduzieren. Würde man die Ausgangsregression ohne die 68 Überschussjahre berechnen, fiele die Differenz sogar noch stärker aus. 
Abbildung 4: Realisierte und prognostizierte Werte für die Entwicklung der Staatstätigkeit
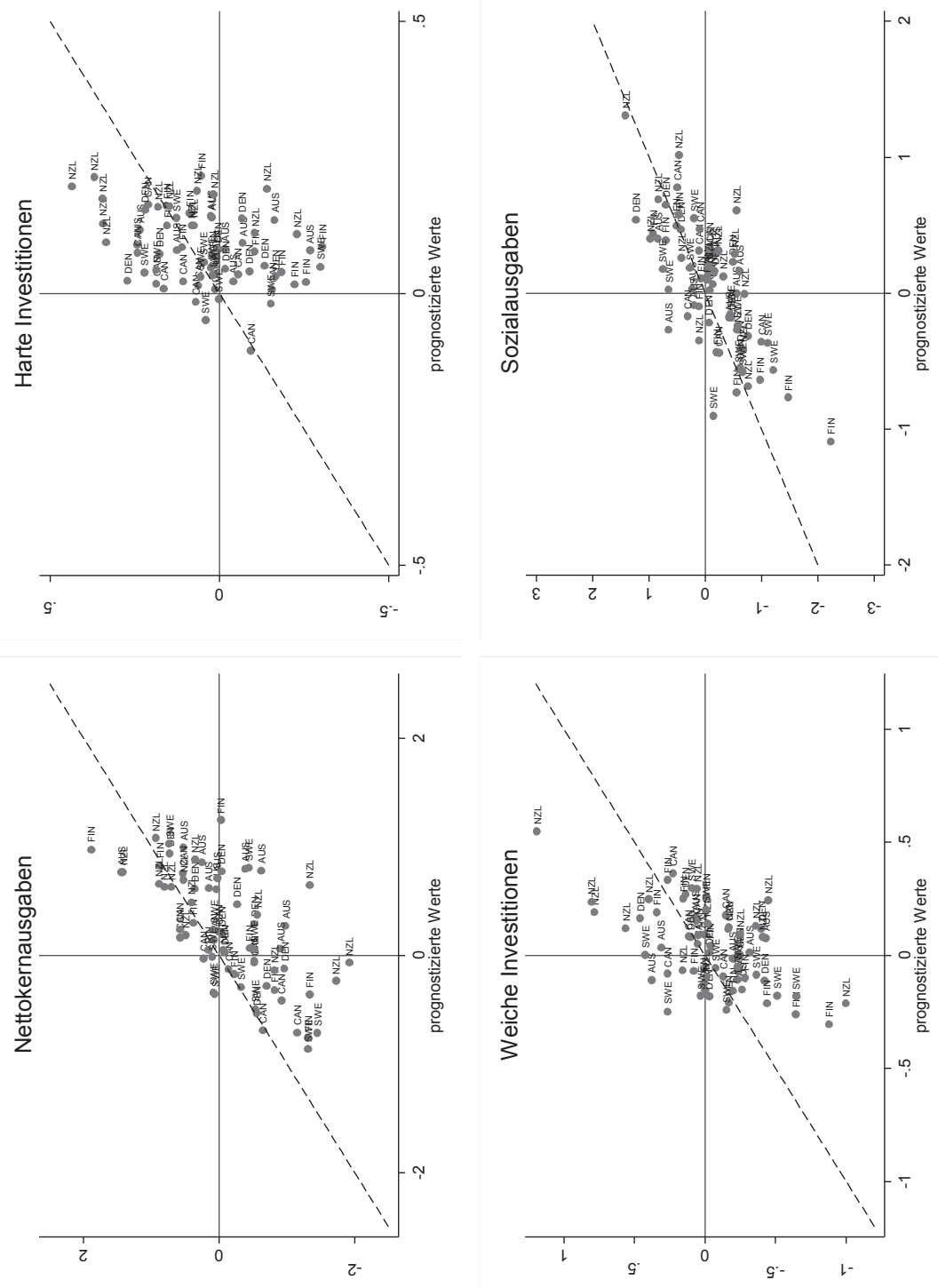

Quelle: Darstellung auf Basis der Regression in Tabelle 5.

Der einzige Bereich, in dem sich die Entwicklung der Defizitjahre im Überschuss tatsächlich umkehrt, sind die zyklisch bereinigten Staatseinnahmen (vgl. Abb. 5), 
wo die Steuern in symmetrischer Weise wieder gesenkt werden. Die Residuen der Staatseinnahmenregression sind nicht von Null zu unterscheiden $(p=0,237)$.

Abbildung 5: Realisierte und prognostizierte Werte für die Entwicklung der zyklisch bereinigten Staatseinnahmen

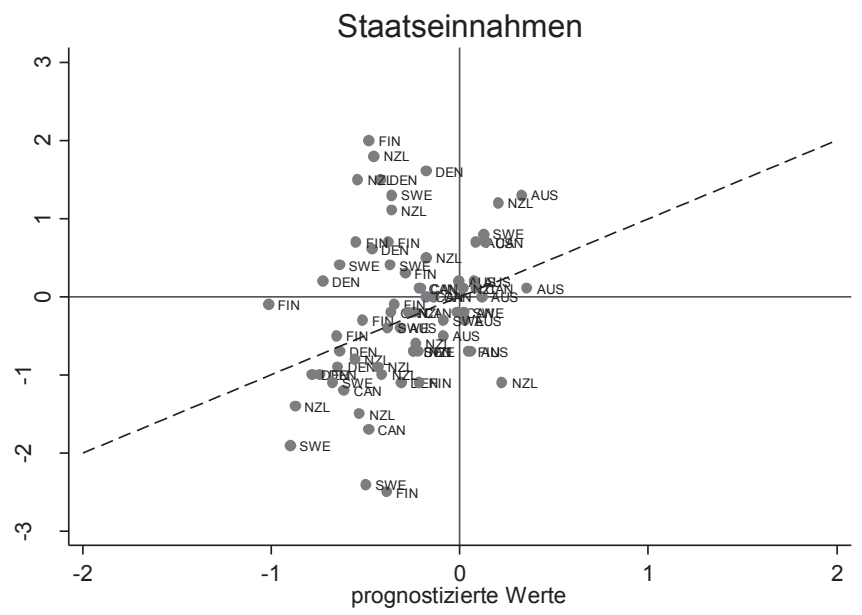

Quelle: Darstellung auf Basis der Regression in Tabelle 5.

Fasst man diese Ergebnisse zusammen, so bestätigen sie die Pfadabhängigkeitsthese in klarer Form: Nur der Bereich staatlichen Handelns, der verhältnismäßig geringe Konsolidierungslasten tragen musste, profitiert in voller Höhe von den Überschüssen. Dagegen fällt den Bereichen, die bereits den Hauptanteil an der Erzielung von Überschüssen hatten, auch die größte Rolle bei ihrer Bewahrung zu. Der unter der Bedingung einer Bewahrung des Konsolidierungserfolgs entstehende Zielkonflikt zwischen den verschiedenen Möglichkeiten fiskalpolitischer Aktivität wird also in derselben Weise gelöst, wie bereits in der Konsolidierung selbst. Ein Politikwechsel findet nicht statt.

Die grafische Analyse der Residuen zeigt zudem, dass diese Ergebnisse nicht von einzelnen Ausreißern getrieben werden, sondern repräsentativ für alle Fälle sind. In praktisch allen Ländern liegt der Schwerpunkt der Punktewolken in Abbildung 4 unterhalb der Geraden, auf der realisierte und prognostizierte Werte einander entsprechen. Die klare Ausnahme ist jedoch Neuseeland, wo sich sowohl bei den harten als auch bei den weichen Investitionen deutliche Ausreißer nach oben zeigen. Ein Grund für diese Besonderheit ist schlicht der enorm hohe Bedarf an Er- 
satzinvestitionen. Neuseeland hatte traditionell den größten öffentlichen Kapitalstock der gesamten OECD und entsprechend hohe Abschreibungen zu tätigen. ${ }^{41}$ Hinzu kam, dass die Erhöhung der Investitionen ein politisches Kernanliegen der seit 1999 regierenden Labour-Regierung war. Zugleich erhöhte diese jedoch auch den Spitzensteuersatz, um ihre politischen Projekte zu finanzieren. ${ }^{42}$ Die höheren Einnahmen stellten sicher, dass der Überschuss trotz des ab 2002 beginnenden Anstiegs der Investitionsquote um mehr als $1 \%$ des BIP nicht in Gefahr geriet.

Tabelle 6: Regressionsresiduen in Überschussjahren mit progressiven Regierungen

\begin{tabular}{l|c|c}
\hline Maßzahl & Durchschnittliches Residuum & p-Wert \\
\hline Nettokernausgaben & $-0,204$ & 0,012 \\
Sozialausgaben & $-0,119$ & 0,101 \\
Weiche Investitionen & $-0,076$ & 0,114 \\
Harte Investitionen & $-0,048$ & 0,067 \\
Zyklisch ber. Einnahmen & 0,203 & 0,202 \\
\hline
\end{tabular}

Die neuseeländische Labour-Regierung verfolgte also tatsächlich einen Politikwechsel, indem sie die Einnahmenseite an der Bewahrung der Konsolidierungserfolge beteiligte und zugleich behutsam die Investitionsausgaben erhöhte. Damit steht sie im internationalen Vergleich jedoch allein. Das gilt insbesondere auch für andere sozialdemokratische oder progressive Parteien. Diese regierten in immerhin 41 der 68 hier untersuchten Überschussjahre. Beschränkt man die Analyse der Residuen auf diese 41 Jahre, so zeigt sich dass die Entwicklung der Staatsausgaben ebenfalls spürbar hinter den Prognosen des Regressionsmodells zurückbleibt (vgl. Tab. 6). Offenbar hat die Lockerung der fiskalischen Restriktionen es den progressiven Parteien nicht ermöglicht, nach einer erfolgreichen Konsolidierung wieder eine dezidiert progressive Politik zu verfolgen. Dieses quantitative Ergebnis stimmt auch mit den Befunden detaillierter Einzelfallstudien zu Ländern wie Kanada $^{43}$, Schweden $^{44}$ und Australien ${ }^{45}$ überein.

41 Kamps, a.a.O. schätzt, dass der Nettokapitalstock des neuseeländischen Staates trotz der Erhöhung der Bruttoinvestitionen zwischen 1990 und 2001 von $102 \%$ auf $75 \%$ des BIP zurückging.

42 Broome, A.: Setting the Fiscal Policy Agenda: Economic News and Election Year Tax Debates in New Zealand, in: Law in Context 24/2 (2006), 60-77, hier 68.

43 Lewis, a.a.O.

44 Gmeiner, J.: Die "Zukunftspartei" Schwedens? Die Schwedische Sozialdemokratie zwischen erfolgreicher Vergangenheit und unsicherer Zukunft, Berlin, 2001; Haffert, L./Mehrtens, P.: From Austerity to Expansion? Consolidation, Budget Surpluses, and the Decline of Fiscal Capacity, in: Politics \& Society, im Erscheinen.

45 Battin, T.: The Australian Labor Party and the Notion of Economic Management, in: Journal of Economic and Social Policy 6/2 (2002), 1-12; Walter, R./Uhr, J.: Budget Talk: Rhetorical Constraints and Contests, in: Australian Journal of Political Science, $48 / 4$ (2013), 431-444. 
Den sechs in dieser Studie untersuchten Ländern gelang es, ihre Konsolidierungserfolge dauerhaft zu bewahren. Der Preis, den sie dafür zahlten war jedoch ein Verzicht auf jenen Politikwechsel, der die Konsolidierung ursprünglich einmal motiviert hatte. Die Ausgaben für staatliche Gestaltungsaufgaben wurden wesentlich langsamer erhöht, als dies bei einer symmetrischen Umkehrung der Zusammenhänge aus dem Defizit zu erklären wäre. Dieser Befund lässt sich auch nicht mit makroökonomischen oder anderen Kontextbedingungen erklären. Zudem gilt dieses Ergebnis nicht nur für konservative, sondern auch für progressive Regierungen: Der erhoffte Politikwechsel ist unabhängig von der Parteizugehörigkeit der Regierung weitgehend ausgeblieben. ${ }^{46}$

Tabelle 7: Regressionsresiduen in Jahren kurzfristiger Überschüsse

\begin{tabular}{l|c|c}
\hline Maßzahl & Durchschnittliches Residuum & p-Wert \\
\hline Nettokernausgaben & $-0,086$ & 0,666 \\
Sozialausgaben & $-0,011$ & 0,881 \\
Weiche Investitionen & 0,022 & 0,770 \\
Harte Investitionen & 0,003 & 0,952 \\
Zyklisch ber. Einnahmen & 0,662 & 0,000 \\
\hline
\end{tabular}

Wie eng dieser Befund mit der Entscheidung zur Bewahrung von Überschüssen zusammenhängt, zeigt abschließend ein Blick auf jene Fälle, die ihren Überschuss nicht bewahren konnten. ${ }^{47}$ Auch für diese werden im Folgenden die Regressionsresiduen untersucht (vgl. Tab. 7). Dabei zeigt sich, dass diese sehr viel stärker mit den Ergebnissen der Analyse für den gesamten Datensatz übereinstimmen. Mit anderen Worten: Die entscheidende Zäsur innerhalb des Datensatzes liegt nicht zwischen Ländern mit Überschüssen und Ländern mit Defiziten, sondern zwischen den Ländern, die ihren Überschuss dauerhaft bewahren konnten und allen übrigen Ländern.

Daneben zeigt Tabelle 7 aber auch, wie sehr es sich bei diesen kurzen Überschussperioden um konjunkturelle Sonderphänomene handelte. Dies wird am gewaltigen Anstieg der Staatseinnahmen sichtbar, bei denen das konjunkturelle Bereinigungsverfahren hier versagt: Diese Einnahmen waren nämlich keineswegs permanenter Natur, sondern vor allem günstigen makroökonomischen Rahmenbedingungen, wie z.B. dem Internetboom der 1990er-Jahre, zu verdanken. Hinter diesen kurzen

46 Eine genauere Untersuchung der konkreten Pfadabhängigkeitsmechanismen hinter diesem quantitativen Ergebnis kann in der vorliegenden Studie aus Platzgründen nicht geleistet werden. Für eine historischinstitutionalistische Analyse der konkreten Pfadabhängigkeiten siehe allgemein Haffert, L., a.a.O.; sowie besonders zum schwedischen Fall Mehrtens, P., a.a.O.

47 Dabei schließen wir die Schweiz aus, deren Überschuss am Ende des Untersuchungszeitraums noch andauerte. 
Überschüssen stand also mindestens ebenso sehr ein Wirtschaftsaufschwung wie politischer Konsolidierungswille. Dagegen handelt es sich bei den sechs ausführlich untersuchten Überschüssen tatsächlich klar um das Ergebnis tiefgreifender politischer Konsolidierungsbemühungen, wie der Blick auf die Entwicklung von Staatseinnahmen und Staatsausgaben im Vorfeld des Überschusses gezeigt hat. Diese mit diesen tiefen Einschnitten verbundenen Maßnahmen prägten noch viele Jahre später die Verwendung der Überschüsse und verhinderten einen progressiven Politikwechsel.

\section{Ein Ausblick auf mögliche deutsche Haushaltsüberschüsse}

Die Frage nach der Verwendung von Haushaltsüberschüssen ist gerade in Deutschland von besonderer Aktualität. So will die Bundesregierung laut Haushaltsentwurf für 2015 vom kommenden Jahr an Überschüsse erzielen. ${ }^{48}$ Bevor diese Überschüsse überhaupt Realität geworden sind, werden bereits von vielen Seiten Forderungen erhoben, wie sie zu verteilen seien. Dabei rücken insbesondere die öffentlichen Investitionen in den Vordergrund. Ein Beispiel unter vielen bieten die großen Wirtschaftsforschungsinstitute, die in ihrer Gemeinschaftsdiagnose im Herbst 2013 forderten, zukünftige Überschüsse dazu einzusetzen, die kalte Progression abzubauen und höhere Investitionen in Infrastruktur, Bildung und Forschung zu tätigen. ${ }^{49}$ Dabei schätzten sie den Bedarf an zusätzlichen Bildungsinvestitionen auf 16 Mrd. Euro und den Bedarf an Infrastrukturinvestitionen auf 8 Mrd. Euro pro Jahr.

Die oben präsentierten Analysen weisen darauf hin, dass die Umsetzung solcher Forderungen auf erhebliche Hindernisse stoßen wird, weshalb mögliche deutsche Haushaltsüberschüsse kein Anlass für übertriebene Erwartungen sein sollten. Diese Interpretation unterliegt allerdings zwei Einschränkungen. Zunächst einmal ist noch völlig unklar, ob diese Überschüsse mehr als eine Episode bleiben werden. Wie gesehen, müssen Überschüsse keine langfristige Entspannung der öffentlichen Finanzlage bedeuten sondern können Teil eines fortbestehenden DefizitKonsolidierungs-Zyklus sein. Sollen die Überschüsse dauerhaft bewahrt werden, wird dies eine anhaltende Selbstbeschränkung der Fiskalpolitik verlangen. Ob Deutschland zu dieser Selbstbeschränkung bereit sein wird, ist schwer zu prophezeien.

Bundesministerium der Finanzen 2014: Bundeskabinett beschließt Haushaltsentwurf 2015, Pressemitteilung vom 02.07.2014.

49 Projektgruppe Gemeinschaftsdiagnose: Konjunktur zieht an - Haushaltsüberschüsse sinnvoll nutzen, Gemeinschaftsdiagnose Herbst 2013. 
Selbst wenn dies der Fall sein sollte, unterscheiden sich die deutschen Überschüsse in verschiedener Hinsicht von den hier untersuchten Fällen. So sind sie weniger harten Sparmaßnahmen, sondern zu großen Teilen konjunkturell bedingten hohen Steuereinnahmen und fallenden Zinsen zu verdanken. Während die sechs untersuchten Überschussländer vor der erfolgreichen Konsolidierung ihrer Haushalte mit einschneidenden Verschuldungskrisen konfrontiert wurden, ${ }^{50}$ ging den deutschen Überschüssen keine Krise der Staatsfinanzen voraus, wenngleich die Schuldenkrisen verschiedener Mitglieder des Euroraums eine bedeutende Rolle spielen dürften. Zugleich fehlt angesichts der in Berlin regierenden großen Koalition aus CDU/CSU und SPD eine politisch gewichtige Opposition, die eine Abweichung vom Überschusskurs politisieren könnte. Andererseits sind, in Übereinstimmung mit den sechs hier untersuchten Ländern, mit der Aufnahme der Schuldenbremse ins Grundgesetz bereits institutionelle Schritte zur Tabuisierung von Defiziten unternommen worden - und zwar in einer Form, die das Ziel einer stärkeren Investitionsorientierung explizit nicht berücksichtigt. ${ }^{51}$

Trotz dieser Unterschiede bieten die Ergebnisse der vorliegenden Studie einigen Anlass zur Skepsis bezüglich der Aussichten einer substantiellen Erhöhung der staatlichen Investitionstätigkeit. So gelang es keinem Land, einerseits die Ergebnisse permanenter Austeritätspolitik - allen voran dauerhaft niedrige Investitionen - zu vermeiden, andererseits aber nicht wieder in einen Zyklus aus Defiziten und Defizitbekämpfung zu geraten. Am nächsten kam diesem Ziel noch Neuseeland, allerdings erst nach besonders drastischen Einschnitten in der Konsolidierung und nach einer Erhöhung der Einkommensteuer. Es wäre bemerkenswert, wenn ausgerechnet Deutschland eine Stärkung der öffentlichen Investitionen bei gleichzeitigem Schuldenabbau gelingen würde. Dies wäre wohl nur möglich, wenn die dafür nötigen Mittel strukturell auf der Einnahmenseite generiert werden könnten und damit ist bis auf weiteres nicht zu rechnen. Stattdessen plant der Bund jetzt die stärkere Beteiligung privater Anleger an öffentlichen Investitionen. ${ }^{52}$

Der neuseeländische Weg, steigende Investitionen bei gleichzeitiger Bewahrung ausgeglichener Haushalte durch Steuererhöhungen zu finanzieren, wurde im Wahlkampf des Jahres 2013 von den Parteien der linken Mitte angeboten. Sie wurde jedoch in den folgenden Koalitionsverhandlungen schnell verworfen. Zwar

50 Baldacci, E./Petrova, I./Belhocine, N./Dobrescu, G./Mazraani, S.: Assessing Fiscal Stress, IMF Working Paper 11/100, 2008.

51 Feld, L.: Sinnhaftigkeit und Effektivität der deutschen Schuldenbremse, in: Perspektiven der Wirtschaftspolitik 11/3 (2010), 226-245, hier 235.

52 Schäuble, W.: Bundeshaushalte ohne Neuverschuldung sollen ab 2015 Normalität werden. Rede des Finanzministers zur Einbringung des Bundeshaushalts vor dem deutschen Bundestag am 09.09.2014. 
verpflichtete sich die neue Regierung dennoch zu einer Erhöhung der öffentlichen Investitionen, diese wird jedoch viel zu klein ausfallen, um den enormen Bedarf an Investitionen in die Infrastruktur zu decken. Der im März 2014 vom Kabinett beschlossene Finanzplan sieht für den Zeitraum von 2014-2018 eine kaum mehr als symbolische Erhöhung der Bruttoinvestitionen von 25,8 auf 27 Mrd. Euro vor. ${ }^{53}$ Stattdessen war die mit Abstand kostspieligste Entscheidung der großen Koalition das von Experten vielfach kritisierte aber in der Bevölkerung sehr populäre Rentenpaket. Hinzu kommt, dass für einen Großteil der Bildungsausgaben und der Investitionen die Länder und Gemeinden verantwortlich sind. Die Vorgaben der Schuldenbremse beschränken die Länder noch stärker als den Bund. ${ }^{54}$ Und hinter dem aggregierten Überschuss der Gemeinden verbirgt sich ein enormes Ungleichgewicht zwischen finanzstarken und finanzschwachen Kommunen.

Es ist deshalb unwahrscheinlich, dass gerade Deutschland gelingt, was allen anderen entwickelten Industrieländern misslang, nämlich dauerhaft ausgeglichene Haushalte mit einer substantiellen Wiedergewinnung staatlicher Handlungsfähigkeit zu verbinden. Fiskalpolitische Handlungsfähigkeit setzt nämlich sowohl die fiskalischen Möglichkeiten als auch den politischen Willen voraus, einen aktiven Staat mit dem Anspruch auf gesellschaftliche Gestaltung zu finanzieren. Solange dieser Wille fehlt, oder sich nicht gegen die Kombination von Steuersenkungen und Ausgabenkürzungen behaupten kann, sind Haushaltsüberschüsse weniger der Ausdruck wachsender Gestaltungsfreiheit des deutschen Staates, als vielmehr ein Ausdruck seiner abnehmenden Gestaltungsambitionen.

54 Während dem Bund ab 2016 noch eine Nettokreditaufnahme von $0,35 \%$ des BIP erlaubt ist, gilt für die Länder ab 2020 ein generelles Verschuldungsverbot. 


\section{Anhang}

Tabelle A1: Residuen für verschiedene Modifikationen der originalen Regressionsspezifikation

\begin{tabular}{|c|c|c|c|c|c|}
\hline & $\begin{array}{l}\text { Nettokern- } \\
\text { ausgaben }\end{array}$ & $\begin{array}{l}\text { Sozialaus- } \\
\text { gaben }\end{array}$ & $\begin{array}{l}\text { Weiche In- } \\
\text { vestitionen }\end{array}$ & $\begin{array}{l}\text { Harte In- } \\
\text { vestitionen }\end{array}$ & Einnahmen \\
\hline \multicolumn{6}{|c|}{ Originale Spezifikation } \\
\hline $\begin{array}{l}\text { Residuen } 6 \text { langer } \\
\text { Überschüsse }\end{array}$ & $\begin{array}{c}-0,365^{* * *} \\
(0.000)\end{array}$ & $\begin{array}{l}-0,133^{*} \\
(0,016)\end{array}$ & $\begin{array}{l}-0,071 \\
(0,051)\end{array}$ & $\begin{array}{l}-0,041^{*} \\
(0,036)\end{array}$ & $\begin{array}{c}0,132 \\
(0,237)\end{array}$ \\
\hline $\begin{array}{l}\text { Residuen progres- } \\
\text { siver Regierungen }\end{array}$ & $\begin{array}{l}-0,204^{*} \\
(0,012)\end{array}$ & $\begin{array}{l}-0,119 \\
(0,101)\end{array}$ & $\begin{array}{l}-0,076 \\
(0,114)\end{array}$ & $\begin{array}{l}-0,048 \\
(0,067)\end{array}$ & $\begin{array}{c}0,203 \\
(0,202)\end{array}$ \\
\hline $\begin{array}{l}\text { Residuen } 10 \text { kur- } \\
\text { zer Überschüsse }\end{array}$ & $\begin{array}{l}-0,086 \\
(0,666)\end{array}$ & $\begin{array}{l}-0,011 \\
(0,881)\end{array}$ & $\begin{array}{c}0,022 \\
(0,770)\end{array}$ & $\begin{array}{c}0,003 \\
(0,952)\end{array}$ & $\begin{array}{c}0,662 * * * \\
(0,000)\end{array}$ \\
\hline \multicolumn{6}{|c|}{ Spezifikation mit fixen Zeiteffekten } \\
\hline $\begin{array}{l}\text { Residuen } 6 \text { langer } \\
\text { Überschüsse }\end{array}$ & $\begin{array}{c}-0,374 * * * \\
(0.000)\end{array}$ & $\begin{array}{l}-0,069 \\
(0,067)\end{array}$ & $\begin{array}{l}-0,071 \\
(0,064)\end{array}$ & $\begin{array}{l}-0,029 \\
(0,156)\end{array}$ & $\begin{array}{l}-0,035 \\
(0,728)\end{array}$ \\
\hline $\begin{array}{l}\text { Residuen progres- } \\
\text { siver Regierungen }\end{array}$ & $\begin{array}{c}-0,203 * * \\
(0,004)\end{array}$ & $\begin{array}{l}-0,113 \\
(0,105)\end{array}$ & $\begin{array}{l}-0,080 \\
(0,107)\end{array}$ & $\begin{array}{l}-0,038 \\
(0,150)\end{array}$ & $\begin{array}{c}0,040 \\
(0,765)\end{array}$ \\
\hline $\begin{array}{l}\text { Residuen } 10 \text { kur- } \\
\text { zer Überschüsse }\end{array}$ & $\begin{array}{l}-0,042 \\
(0,824)\end{array}$ & $\begin{array}{l}-0,001 \\
(0,991)\end{array}$ & $\begin{array}{c}0,055 \\
(0,473)\end{array}$ & $\begin{array}{c}0,001 \\
(0,983)\end{array}$ & $\begin{array}{c}0,613 * * * \\
(0,000)\end{array}$ \\
\hline \multicolumn{6}{|c|}{ Spezifikation mit Dekadendummys für die 80er-und 90er-Jahre } \\
\hline $\begin{array}{l}\text { Residuen } 6 \text { langer } \\
\text { Überschüsse }\end{array}$ & $\begin{array}{c}-0,380 * * * \\
(0.000)\end{array}$ & $\begin{array}{l}-0,132 * \\
(0,017)\end{array}$ & $\begin{array}{l}-0,074 \\
(0,051)\end{array}$ & $\begin{array}{l}-0,036 \\
(0,065)\end{array}$ & $\begin{array}{c}0,113 \\
(0,314)\end{array}$ \\
\hline $\begin{array}{l}\text { Residuen progres- } \\
\text { siver Regierungen }\end{array}$ & $\begin{array}{c}-0,210 * * \\
(0,009)\end{array}$ & $\begin{array}{l}-0,121 \\
(0,098)\end{array}$ & $\begin{array}{l}-0,073 \\
(0,131)\end{array}$ & $\begin{array}{l}-0,043 \\
(0,097)\end{array}$ & $\begin{array}{c}0,183 \\
(0,251)\end{array}$ \\
\hline $\begin{array}{l}\text { Residuen } 10 \text { kur- } \\
\text { zer Überschüsse }\end{array}$ & $\begin{array}{l}-0,065 \\
(0,741)\end{array}$ & $\begin{array}{l}-0,014 \\
(0,845)\end{array}$ & $\begin{array}{c}0,019 \\
(0,808)\end{array}$ & $\begin{array}{l}-0,004 \\
(0,936)\end{array}$ & $\begin{array}{c}0,690 * * * \\
(0,000)\end{array}$ \\
\hline \multicolumn{6}{|c|}{ Spezifikation mit Country-Fixed Effects } \\
\hline $\begin{array}{l}\text { Residuen } 6 \text { langer } \\
\text { Überschüsse }\end{array}$ & $\begin{array}{c}-0,180^{* *} \\
(0,009)\end{array}$ & $\begin{array}{l}-0,074 \\
(0,160)\end{array}$ & $\begin{array}{l}-0,074 \\
(0,050)\end{array}$ & $\begin{array}{l}-0,012 \\
(0,521)\end{array}$ & $\begin{array}{c}0,064 \\
(0,545)\end{array}$ \\
\hline $\begin{array}{l}\text { Residuen progres- } \\
\text { siver Regierungen }\end{array}$ & $\begin{array}{l}-0,147 \\
(0,102)\end{array}$ & $\begin{array}{l}-0,084 \\
(0,210)\end{array}$ & $\begin{array}{l}-0,076 \\
(0,110)\end{array}$ & $\begin{array}{l}-0,033 \\
(0,499)\end{array}$ & $\begin{array}{c}0,028 \\
(0,851)\end{array}$ \\
\hline $\begin{array}{l}\text { Residuen } 10 \text { kur- } \\
\text { zer Überschüsse }\end{array}$ & $\begin{array}{l}-0,388^{*} \\
(0,048)\end{array}$ & $\begin{array}{l}-0,042 \\
(0,539)\end{array}$ & $\begin{array}{c}0,031 \\
(0,649)\end{array}$ & $\begin{array}{l}-0,008 \\
(0,718)\end{array}$ & $\begin{array}{c}0,749 * * * \\
(0,000)\end{array}$ \\
\hline
\end{tabular}

